
\title{
$\begin{array}{ll}\text { Research Square } & \text { They should not be considered conclusive, used to inform clinical practice, } \\ \text { or referenced by the media as validated information. }\end{array}$
}

\section{A highly efficient cloth facemask}

Christopher Cappa ( $\square$ cdcappa@ucdavis.edu )

University of California, Davis https://orcid.org/0000-0002-3528-3368

The San Francisco Opera Costume Department

The San Francisco Opera

William Ristenpart

University of California, Davis

\section{Santiago Barreda}

University of California, Davis

Nicole Bouvier

Icahn School of Medicine

Elad Levintal

University of California, Davis

Anthony Wexler

University of California, Davis https://orcid.org/0000-0003-1565-814X

\section{Sanziana Roman}

University of California, San Francisco

\section{Research Article}

Keywords: face masks, face coverings, respiratory disease, public health, aerosols, airborne

Posted Date: July 27th, 2021

DOl: https://doi.org/10.21203/rs.3.rs-322107/v3

License: (9) (1) This work is licensed under a Creative Commons Attribution 4.0 International License. Read Full License 


\title{
Title: A highly efficient cloth facemask design
}

Authors: Christopher D. Cappa ${ }^{1, *}$, The San Francisco Opera Costume Department ${ }^{2, \dagger}$, William D. Ristenpart, ${ }^{3}$ Santiago Barreda, ${ }^{4}$ Nicole M. Bouvier, ${ }^{5}$ Elad Levintal, ${ }^{6}$ Anthony S. Wexler, ${ }^{7}$ Sanziana A. Roman ${ }^{8, *}$

Affiliations:

${ }^{1}$ Dept. of Civil and Environmental Engineering, Univ. of California Davis; 1 Shields Ave., Davis, CA 95616 USA

${ }^{2}$ Costume Department, San Francisco Opera; 301 Van Ness Ave, San Francisco, CA 94102, USA

${ }^{3}$ Dept. of Chemical Engineering, Univ. of California Davis; 1 Shields Ave., Davis, CA 95616 USA.

${ }^{4}$ Dept. of Linguistics, Univ. of California Davis; 1 Shields Ave., Davis, CA 95616 USA.

${ }^{5}$ Department of Medicine, Division of Infectious Diseases, Icahn School of Medicine at Mount Sinai, 1 Gustave L. Levy Place, New York, NY 10029, USA

${ }^{6}$ Dept. of Land, Air and Water Resources, University of California, Davis, 1 Shields Ave., CA 95616 USA.

${ }^{7}$ Dept. of Mechanical Engineering, Univ. of California Davis; 1 Shields Ave., Davis, CA 95616 USA.

${ }^{8}$ Department of Surgery, Univ. of California, San Francisco; 1660 Divisadero St., San Francisco, CA 94143 USA

${ }^{\dagger}$ Includes: Amy Ashton-Keller, Daniele McCartan, Galen Till, Jai Alltizer

*Corresponding authors: cdcappa@ucdavis.edu or Sanziana.Roman@ucsf.edu

\begin{abstract}
Wearing masks and face coverings helps reduce transmission of respiratory diseases. Much prior research on mask filtration efficiency has focused on fabric type, with less consideration given to mask design and shape. Here, we present evidence that increasing the mouth-mask separation distance engenders an increase in the mask-fabric filtration efficiency towards expiratory aerosols emitted via vocalization, which we attribute to a decrease in the expiratory jet velocity and expansion of the breath-mask contact area. We further assess the performance of a new reusable cloth facemask design, originally made for, but not exclusive to, professional singers and having a large mouth-mask separation distance. With proper fitting, these masks achieve overall filtering efficiencies of $>93 \%$ for both exhaled expiratory aerosols $>0.5$ microns in diameter from singing and speaking and for inhaled ambient aerosols, better than surgical masks and approaching N95 respirators. Air exchange between the mask deadspace and the ambient environment limits the extent of $\mathrm{CO}_{2}$ buildup and in inhaled air and maintains $\mathrm{O}_{2}$ levels near ambient. The mask design also provides for less inhibited mouth and jaw movement and lower relative humidity, suggesting it may provide a more palatable, high-efficiency alternative to medical-grade masks for the public.
\end{abstract}




\section{One Sentence Summary}

A new cloth mask design originally made for singing provides high filtration of respiratory and ambient aerosols.

\section{Short Title}

A highly efficient cloth facemask design

\section{Introduction}

Respirators, face masks, and coverings play important roles in protecting wearers in various occupational settings and are important public health tools for reducing transmission of respiratory disease [Brooks and Butler, 2021; Howard et al., 2021]. Masks differ in style, features, materials, and fit - including their overall level of protection - to account for different exposure conditions and wearer requirements. Often the most high-efficiency masks, such as N95 respirators, are those in the least supply, especially in times of critical need such as during the COVID-19 pandemic [Dai et al., 2020]. When particularly transmissible variants of respiratory diseases arise, as is currently the case with the SARS-CoV-2 virus [Chand et al., 2021], the need grows for masks for the public having high efficiency, but that have distinct supply chains from medical-grade masks and respirators.

Willingness to wear facemasks depends on real and perceived psychological and physiological drawbacks of mask wearing, which includes increased skin temperature, elevated humidity, difficulty breathing, and discomfort when speaking [Scheid et al., 2020; Taylor and Asmundson, 2020]. New mask designs that help to mitigate these drawbacks may precipitate greater willingness of people to wear masks, especially if such masks have high overall filtration efficiencies. Most prior research has focused on the tradeoff between filtration efficiency and breathability, with less porous materials providing more filtration and less facile breathing [Bagheri et al., 2021; Drewnick et al., 2021; Shakya et al., 2017]. Less attention has focused on the overall shape of the mask; the vast majority of mask designs have the fabric in immediate proximity to the mouth.

A notable exception involves a variety of facemask originally developed by singers. These masks feature a very large internal space, with the mouth situated several centimeters away from the fabric (Figure 1). Singing and theater performing require mask designs that generally allow for 
much more jaw movement compared to other professions as well as increased space between the face and the mask material. Thus, any mask appropriate for singing will also be more comfortable for speech, generally. Additionally, group singing presents a particular challenge for community transmission of respiratory disease owing to multiple people vocalizing consistently and at the same time [Miller et al., 2021] (compared to speaking, where people typically take turns) and the loudness of singing compared to normal speech, as the production of potentially virus-laden aerosols increases with volume [Alsved et al., 2020; Asadi et al., 2019]. To date, however, there has been no characterization of the overall filtration efficiency of these masks for either exhaled respiratory or inhaled ambient aerosols nor to assess the importance of the generally increased mouth-mask fabric distance for these masks compared to other designs.

Here, we characterize the reduction efficiency towards exhaled respiratory aerosols alongside $\mathrm{CO}_{2}$ buildup and $\mathrm{O}_{2}$ depletion of one such mask designed originally for singing, but that may be of use for wearing by the public. We also explicitly characterize the impact of varying the mouthmask distance on exhaled respiratory aerosol filtration efficiency. The "singing mask" here (Figure 1a,b) allows for facile jaw movement compared to conventional masks tested to date yet maintains overall high efficiency towards emission of expiratory aerosols, even after accounting for leakage flows. The singing mask here shares some similarities with other masks targeting singing that have been introduced during the COVID-19 pandemic, but differs from these others in terms of fit, material, and adjustability. We also characterize the mask efficiency for filtration of ambient particles, demonstrating substantial protection to the infection-naive wearer as well. Although designed to accommodate singers, the high overall mask efficiency (>93\%) suggests that the singing mask can provide a useful alternative to existing high-efficiency masks (e.g., N95's) for the public.

\section{Materials and Methods}

\subsection{Mask Design}

The singing mask, shown in Figure 1, uses a two-bone structure to separate the mask material from the main area of the face by about $6 \mathrm{~cm}$, while still allowing for a good seal. The mask was

developed by co-author S. A. R. in partnership with the San Francisco Opera. A $0.6 \mathrm{~cm}$ wide, 10 $\mathrm{cm}$ long thin aluminum strip is used around the nose, which the wearer can mold to their face. A felt strip on the inside runs across the nose area to help with sealing. The sides of the mask extend 
over the cheeks, nearly to the ears. Adjustable elastic ear loops keep the sides of the mask in place and two additional ties fasten the mask around the wearer's head to further seal the mask against the face. The mask completely envelops the wearer's jaw and chin, with an adjustable elastic band below the jaw that keeps the mask tight against the neck while allowing for free jaw movement. The mask has two main regions: the upper, boned structure that holds the filtering fabric in front of but away from the mouth and nose, and an unstructured, expanded volume below the chin. The upper region is composed of three layers in a cloth-liner-cloth arrangement, with 200 thread count cloth outer layers and a Pellon ${ }^{\circledR} 50$ (Pellon Consumer Products, Saint Petersburg, FL) inner layer attached to the cotton with a fusible webbing material. The Pellon ${ }^{\circledR} 50$ inner layer helps to stiffen the mask material but likely provides little filtering. The length of the top region is about $12 \mathrm{~cm}$. The lower expanded volume is made of two cloth layers and opens at the bottom to allow for drinking by straw during rehearsals and other situations where drinking occurs (e.g., schools, cafes, bars), which could lower the risk of exposure compared to removing the mask. The opening is sealed by folding the mask twice and then securing with embedded Velcro strips. When closed, the length of the expanded region is about $10 \mathrm{~cm}$. A modified version of the singing mask was also constructed. The difference from the standard singing mask is that there is no bottom opening; the modified mask is otherwise identical. During use, the cloth material comprising both the singing and modified singing masks was observed to deflect inward (for inhalation) and outward (for exhalation). However, the boning provides support that limits the amount of cloth deflection associated with inhalation and exhalation. With intentionally loose wearing, the deflection magnitude decreased. Hence, deflection provides a qualitative indication of good fit. The mask internal volume is about $0.5 \mathrm{~L}$. This is similar to the tidal volume associated with normal breathing [Hallett et al., 2020], but about half that for singing [Binazzi et al., 2006]. The mask internal volume is about 3-5 times larger than that for N95 respirators [Xu et al., 2015]. Given the limited deflection of the mask material this implies substantial exchange of air, which will help to alleviate any buildup of $\mathrm{CO}_{2}$ or depletion of $\mathrm{O}_{2}$ (see Section 3.5).

Proper wearing of the singing masks includes: first securing the mask using the ear loops, molding the aluminum strip around the nose, tightening the ear loops, tightening the neck strap elastic band, tying the top strap around the users head near the parietal eminence, and then tying the bottom strap around the users neck while sitting or standing up straight. With proper wearing, one should see no obvious gaps, especially around the nose; this can be qualitatively assessed by 
having the wearer look down towards their nose moving only their eyes. If they can see their nose below the mask then there is a gap and the mask should be better secured.

Two additional masks were constructed using the same materials as the singing mask. One was constructed having only two cloth layers and one having three layers (cloth-liner-cloth). Both used a general pleated surgical mask design, based on the design initially promoted by the U.S. Centers for Disease Control; the directions and instructional video originally made available by the CDC are no longer available online. Both used the same ear loops as with the singing mask and included two head straps. Additional tests for the through-mask efficiency were performed by one participant using an N95 respirator (3M, Model 8210), two different surgical masks (a medicalgrade ValuMax 5130E-SB and an unknown model), and a non-medical 'Fashion Dust Preventive Mask' (30\% cotton, 70\% polyester) from YiWu Xuefeng Mask Factory, both without (FDPM) and with (FDPM(N95)) an N95 insert.

\subsection{Human subjects}

We recruited 12 volunteers ( 4 self-identified male and 8 self-identified female), ranging in age from 18 to 65 years old. The Institutional Review Board of the University of California, Davis approved this work (IRB\# 844369-4), and all research performed followed the Institutional Review Board guidelines and regulations. Prior to the tests, written informed consent was obtained from all participants. Information collected from participants included their age and singing range (e.g., soprano, alto, baritone). Only self-reported healthy non-smokers were included in the study. All participants had to take the UC Davis Daily Symptom Survey (https://campusready.ucdavis.edu/symptom-survey) prior to accessing campus. Participants were encouraged to obtain a negative COVID-19 test just prior to their participation, although this was not required or tracked. Informed consent for publication of identifying information was obtained from the participant shown in Figure 1.

\subsection{Expiratory Aerosol Experimental Description}

We used an experimental setup similar to that in previous work [Asadi et al., 2020a; Asadi et $a l ., 2019 ; 2020 c]$. In brief, participants were asked to breath, speak, or sing in front of a stainless steel funnel ( $7.5 \mathrm{~cm}$ diameter) connected by nonconductive tubing to an aerodynamic particle sizer (APS, TSI Model 3321, $5 \mathrm{~L} / \mathrm{min}$ ) and a condensation particle counter (CPC, TSI Model 3775, 0.3 
lpm) that was located in a HEPA-filtered laminar flow hood (Figure S1a). The APS characterizes particles from 0.3 to 20 microns in aerodynamic diameter in 51 size bins, with a decreased detection efficiency for particles $<0.5$ microns and the smallest size reported as 0.54 microns. The APS total flow is $5 \mathrm{lpm}$, from which $1 \mathrm{lpm}$ is sub-sampled for characterization of the particle concentration and with the other $4 \mathrm{lpm}$ used as a sheath flow. The CPC characterizes the number concentration of all particles sampled, although with a reduced efficiency for particles larger than about 1 micron owing to impaction losses. We focus primarily on the measurements made using the APS and, unless otherwise stated, results for expiratory aerosols use the APS data.

Participants donned the singing mask without direct assistance. They were asked to tighten the ear loops and the neck closure, pinch closed the metal bar in the singing mask around their nose, and to tie the neck and head straps. They were asked to "tighten everything as much as possible, but such that you are still comfortable."

Respiratory emissions with or without a mask were tested with the participant's head oriented in one of four positions, relative to the sampling funnel. These orientations were the same as those described in Cappa et al. [2021] and are shown in Figure S1. These were as follows.

(i) Forward/Through: The participants sat directly facing the APS funnel. This was the orientation examined in prior studies [Asadi et al., 2020a; Asadi et al., 2019; 2020c]. In this orientation, the APS samples air that has passed through the mask material.

(ii) Top: The participants tilted their heads downward to have the bridge of the nose approximately centered on the APS funnel, allowing for sampling of particles that leak from the mask nose area.

(iii) Side: The participants turned their head 90 degrees to face perpendicular to the APS funnel, with the side singing mask approximately centered on the funnel

(iv) Bottom: The participants positioned their chin just above the APS funnel with the mask material from the expanded volume over the top of the funnel. This allowed for sampling of particles that leak from the mask neck area.

Participants performed the speaking and singing activities while either wearing or not wearing the singing mask. Measurements without a mask were made only in the forward direction, with the exception of a few test measurements on one participant to confirm that no particles were 
measured above the background level in non-forward directions. Breathing was performed only with no mask; results are not reported here but were consistent with previous measurements. For speaking, participants were asked to read the entirety of the Rainbow Passage, both with no mask and while wearing the mask while oriented in the "forward" direction (Figure S1); the Rainbow Passage is commonly used in respirator fit tests (albeit, a shortened version [Occupational Safety and Health Administration, 2004]) and linguistics research [Fairbanks, 1960]. Participants also performed two singing activities. First, they sang in English Beethoven's Ode to Joy from his Ninth Symphony, both wearing and not wearing the mask, with the latter in each of four head orientations described above (Figure S1). Second, participants sang a song of their choosing of about two minutes in length. They performed this second activity both without a mask and with the mask in the forward orientation only.

For all speaking and singing activities, participants were asked to carry out the activity at a comfortable volume; no effort was made to control for volume differences between participants. While loudness can influence the emission rate of expiratory aerosols [Asadi et al., 2019], we focus on the reduction achieved by wearing the mask, and thus loudness differences between participants will have little effect. All particle emission rates were adjusted to units of particles per second by accounting for the actual duration of vocalization $\left(t_{\mathrm{voc}}\right)$, which excludes pauses between words or phrases as determined from microphone recordings. One participant repeated the Ode to Joy activities multiple times on different days. Participants generally performed the tasks in the order breathing, speaking, singing Ode to Joy, and then singing the participant-selected song. We varied the order in which the participants performed a given task with or without mask wearing. For singing Ode to Joy with mask wearing the order of sampling in the different orientations varied between participants, although typically with the forward direction activity the first performed (either with or without the mask).

The directly observed particle emission rates $\left(\dot{N}_{p}^{o b s}\right)$ does not necessarily equate to the total particle emission rate owing to differences between the APS total airflow rate $\left(Q_{\text {APS,tot }}=5 \mathrm{lpm}\right)$, sub-sampled airflow rate $\left(Q_{\mathrm{APS}, \mathrm{samp}}=1 \mathrm{lpm}\right)$ in the APS in which particle counts are measured, and the airflow rate of the expiratory activity $\left(Q_{\exp }\right)$, as discussed in Cappa et al. [2021]; we refer readers there for details. In brief, when the sampled expiratory airflow exceeds the APS total flowrate the $\dot{N}_{p}^{o b s}$ underestimates the true particle emission rate $\left(\dot{N}_{p}\right)$ by a factor of $Q_{\text {exp }} / Q_{\text {APS,samp }}$, 
although the measured particle concentration is correct. When the sampled expiratory airflow is less than $Q_{\text {APS,tot }}$ the $\dot{N}_{p}$ is also underestimated owing to dilution, but by a constant ratio ( $\left.Q_{\text {APS,tot }} / Q_{\text {APS,samp }}\right)$, while the particle concentration is underestimated by $Q_{\text {APS,tot }} / Q_{\text {exp. }}$.

This raises certain challenges when combining the measurements from the different orientations to estimate the overall mask efficiency. Typical airflow rates associated with talking range from ca. 8-15 lpm [Gupta et al., 2010]. For singing, airflow rates are in the same general range although skewed perhaps a little higher, especially for louder singing, and females tend to exhibit slightly smaller values than males [Holmberg et al., 1988; Jiang et al., 2016]. Consequently, the actual particle emission rates associated with talking and singing without a mask are about a factor of 8-15 times higher than the observed values (the ratio between the actual expiratory airflow rate and the sub-sampled APS airflow rate).

With mask wearing the airflow during expiration can be split in multiple directions, with the expiratory airflow in a given direction not known a priori. We previously accounted for this split for surgical masks while talking or coughing using a Monte Carlo method that accounts for the above mentioned impact of expiratory airflow rates on the observed emission rates and concentrations; this procedure allowed for determination of probability distributions of the overall mask efficiency based on the median values across the population of participants and various assumptions of how the flows are split [Cappa et al., 2021]. Overall, relatively narrow probability distributions resulted with only moderate sensitivity to the assumed split between the air that passed through the mask versus escaped out the top, sides, or bottom and the greatest deviations found for very low total expiratory airflow rates. We use a similar approach here, but apply the approach to the observations from each individual, rather than using the medians across participants. Over 10,000 iterations, we determined the fraction $\left(f_{\mathrm{x}}\right)$ of air that goes in a particular direction from a random distribution, but constrained such that the concentration (rather than count rate) in any direction is less than or equal to that with no mask wearing. We further assumed a lognormal distribution of expiratory airflow rates centered at $13 \mathrm{lpm}$ with a width of 1.3 . In the case when the airflow rate in a given direction exceeds the APS total airflow the inlet is overflowed

The $\dot{N}_{p, i}^{o b s}$ in each orientation ( $i$ ) for each individual are adjusted to actual particle emission rates $\left(\dot{N}_{p, i}\right)$ based on the above assumptions. While we present the unadjusted (observed) absolute particle emission rates to remain consistent with previous studies, when reporting particle emission 
rates normalized to the no-mask condition we use the airflow-adjusted values, which are also used to calculate the overall mask efficiency. The overall mask efficiency, $\eta$, is:

$$
\eta=1-\frac{\sum \dot{N}_{p, i}}{\dot{N}_{p, \text { nomask }}}
$$

The average value and standard deviation for each individual were determined from the distribution of $\eta$ values from the simulations. For comparison, we also determined individual overall efficiencies for the participants speaking while wearing surgical masks in Cappa et al. [2021].

In the "bottom" orientation, the participants positioned their chin just above the APS funnel, with the mask material from the expanded volume draped over the top of the funnel. Some participants could not completely avoid contact between the mask material and the funnel in this position; consequently, mask fibers shed by friction between mask and funnel may have contributed substantially to the particle counts from participants in this orientation [Asadi et al., 2020a; Clark and Shirley, 1973; Hospodsky et al., 2012]. Shedding of skin or hair can also contribute non-expiratory particles during the speaking and singing activities (Hospodsky et al. [2012] and Supplemental Material). Such non-expiratory particles confound the respiratory emission measurements, but they may still carry pathogens as aerosolized fomites [Asadi et al., 2020b]. Based on qualitative analysis of the observed particle size distributions (Figure S2) coupled with a subjective assessment of the extent of mask-funnel contact during singing, a few participants appeared to generate a significant amount of mask-fiber or, potentially, skin or hair, particles. For these participants, when assessing the overall mask efficiency, we used the median value from the other participants in place of the value measured for the individual, although provide discussion of the impact of using unadulterated measurements.

To address concerns that the directional sampling approach used above might miss some particles that escape from the mask edges owing to the modest flowrate of the APS, we also made measurements for one participant using a larger funnel $(30 \mathrm{~cm}$ diameter) and where an ancillary flow of $25 \mathrm{lpm}$ was pulled from between the funnel neck and APS inlet such that the total flow into the funnel exceeded typical expiratory flow rates. The results from these measurements were consistent with the directional sampling approach and are discussed further in the Supplemental Material (Figure S3 and Table S1). They also point to potentially important contributions of skin 
or hair shedding during all activities, which would lead to an underestimate of the actual filtration efficiencies towards expiratory particles; thus, the reported values might be reasonably considered lower limits.

One participant sang Ode to Joy wearing a variety of mask types (see Mask Design) in the forward (through-mask) position, with three replicates for each mask type.

All data processing analyses were carried out using Igor Pro (v. 8.0.4.2, Wavemetrics).

Differences between the $\dot{N}_{p, i}$ values are calculated after log-transformation using a single factor ANOVA test.

\subsection{Inhalation Experimental Description}

The concentration of particles inside the singing mask was measured for one participant while breathing. For comparison, the concentration of particles in the room air was measured just prior to the measurements of particle concentrations inside the mask. Here, a tube composed of conductive silicon was inserted below the mask at the neck area and the sampling end of the tube was positioned to sit in the main mask area in front of the face. The tube was attached to a condensation particle counter (CPC, TSI Model 3775, $0.3 \mathrm{lpm}$ ), which sampled at $0.3 \mathrm{lpm}$ and measured the total concentration of particles every 1 second. The CPC characterizes the number concentration of all particles sampled above $4 \mathrm{~nm}$, although with a reduced efficiency for particles larger than about 1 micron owing to impaction losses. Two experiments were conducted. In both, the participant was asked to breathe deeply in and out through their nose 10 times at a rate of about five breaths per minute while the particle concentration inside the mask was continually monitored. In one experiment, the neck strap was fully tightened, as appropriate for correct fit of the singing mask. For the second, the neck strap was left slightly loose to intentionally introduce a leak. Prior to starting the measurement, the participant was asked to breathe three times after the sampling tube was inserted. The ratio between the room air concentration and the in-mask particle concentration provides a measure of the mask fit factor and the efficiency towards inhaled ambient particles.

\subsection{Filtration Efficiency versus Distance Experimental Description}

The influence of the mask-mouth separation distance on filter efficiency was characterized as follows. A fibrous filter having moderate efficiency was secured over the APS sampling funnel 
using an elastic band. This provided the filtration material, and the "mask"-mouth separation distance was characterized as the distance between the participants' mouth and the fibrous filter. To eliminate the influence of the air from the laminar flow hood on the measurements, a cylindrical sheath having the same diameter as the APS sampling funnel was constructed out of aluminum foil. The sheath was secured to the APS sampling funnel using a second elastic band to make a seal. The distance that the sheath extended from the APS sampling funnel could be adjusted over the range $1 \mathrm{~cm}$ to $10 \mathrm{~cm}$. A schematic is shown in Figure 2a. Background concentrations were unaffected by the presence of the sheath. Without this sheath, the measured particle concentrations during speaking the Rainbow Passage, and without the fibrous filter, would decrease as the participant moved further from the APS sampling funnel. More specifically, without the sheath the measured concentration was constant within measurement reproducibility when the participant was within $3 \mathrm{~cm}$ of the plane of APS sampling funnel but decreased with further distance. With the sheath (and without the filter), there was no discernible change in the measured concentration with distance.

A participant was asked to recite the Rainbow Passage with the sheath set at distances ranging from 1 to $10 \mathrm{~cm}$. The participant gently placed the bridge of their nose and their chin against the sheath to maintain a given distance throughout the activity. Because the participant's mouth extended $\sim 0.5 \mathrm{~cm}$ into the sheath, the reported distances are $0.5 \mathrm{~cm}$ less than the length of the sheath. The participants face did not entirely cover the sheath. Because the sheath was sealed to the sampling funnel, excess air from speaking (relative to the APS flow) exited by the participants face. The distances were selected in a random order. At each distance three replicates were performed with the fibrous filter in place over the APS sampling funnel and compared to the measurements made with no filter. The expiratory air velocity decreases with distance from the mouth, while the fraction of the filter area through which the expiratory air passes increases with distance (see Appendix A). Expiratory air velocities at the mouth exit depend on the airflow rate and the size of the mouth opening. For reference, Kwon et al. [2012] observed airflow velocities of $20-40 \mathrm{~cm} / \mathrm{s}$ over $1-2 \mathrm{~cm}$ distance in front of speakers mouths, which will decrease to about 2-4 $\mathrm{cm} / \mathrm{s}$ at a distance of $10 \mathrm{~cm}$. These values are notably higher than the face velocity associated with the APS airflow through the filter material $(1.06 \mathrm{~cm} / \mathrm{s})$.

The particular fibrous filter used necessarily exhibited little fiber shedding and also had a moderate overall filtration efficiency. The latter condition is necessary because if the filtration 
efficiency is too large it is difficult to determine changes with distance quantitatively within the measurement uncertainty, and working with a fibrous filter having a moderate ( $50 \%)$ efficiency allows for access of a greater range of values. To meet these requirements, we used the outer layer of a 3-layer surgical mask as the fibrous filter, specifically the outer layer of the ValuMax 5130ESB mask. (The inner layer of this mask had too high of an efficiency. The singing mask, despite limited evidence of substantial shedding when worn by the participants, shed particles excessively when secured over the APS sampling funnel.)

The total filtration efficiency was determined by summing over all particles measured. The size-dependent filtration efficiency was determined in five size bins: the lower size limit to 0.8 $\mu \mathrm{m}, 0.8-1.25 \mu \mathrm{m}, 1.25-1.9 \mu \mathrm{m}, 1.9-3.9 \mu \mathrm{m}$, and 3.9-10 $\mu \mathrm{m}$. Uncertainties were taken as the $1 \sigma$ standard deviation of the mean over the three replicates.

This method of determining the material filtration efficiency differs from standard methods that affix the filter material in a holder and vary the airflow rate to control the face velocity [ASTM International, 2017; Drewnick et al., 2021]. In such tests the face velocity is the same across the entire filter material, whereas here the velocity and exposed area co-vary.

\subsection{Measurement of environmental parameters in the mask deadspace volume}

We measured the steady state concentration of $\mathrm{CO}_{2}$ and $\mathrm{O}_{2}$ in the deadspace volume of the singing mask for one participant using a Sensiron SCD-30 NDIR $\mathrm{CO}_{2}$ sensor and Maxell KE-25 $\mathrm{O}_{2}$ sensor, respectively. The sensors recorded at $2 \mathrm{sec}$ time intervals, although the response times were $20 \mathrm{~s}$ and $15 \mathrm{~s}$, respectively. The $\mathrm{CO}_{2}$ sensor has a specified measurement range up to 40,000 $\mathrm{ppm}_{\mathrm{v}}$, although we found that it maxed out above $\sim 32,000 \mathrm{ppm}_{\mathrm{v}}$. The specified accuracy of the $\mathrm{CO}_{2}$ sensor is $+/-(30 \mathrm{ppm}+3 \%)$ up to $10,000 \mathrm{ppm}_{\mathrm{v}}$ and a repeatability of $10 \mathrm{ppm}_{\mathrm{v}}$, while the $\mathrm{O}_{2}$ sensor accuracy is $+/-1 \%$. We have confirmed linearity of the $\mathrm{CO}_{2}$ sensor response up to 30,000 $\mathrm{ppm}_{\mathrm{v}}$ via comparison with a gas chromatograph $\left(r^{2}=0.999\right.$, slope $=0.92$; Figure S4). The $\mathrm{CO}_{2}$ and $\mathrm{O}_{2}$ concentrations were measured separately to avoid contact between the sensors. The SCD30 sensor also measures relative humidity $(\mathrm{RH})$ and temperature $(T)$, with accuracies of $+/-3 \%$ and $+/-0.4^{\circ} \mathrm{C}$ and repeatabilities of $0.1 \%$ and $0.1{ }^{\circ} \mathrm{C}$, respectively.

Two measurements each were made for speaking the Rainbow Passage and singing Ode to Joy for $\mathrm{CO}_{2}$, along with $\mathrm{RH}$ and $T$. Only one measurement was made for $\mathrm{O}_{2}$ for speaking. However, 
we also measured $\mathrm{O}_{2}$ for one participant over a 30 minute period while they sat quietly working at a computer. The sensors were set inside the singing mask deadspace volume with the wires arranged to exit at the neck area; the wires were sufficiently thin that they did not perturb the mask fit in any notable manner. We also measured steady state $\mathrm{CO}_{2}$ in the deadspace of a KN95 respirator worn by one participant while speaking. The smaller deadspace volume led to unavoidable contact between the sensor and the participants face or the respirator, which caused occasional false negative signals that quickly recovered; these negative signals have been removed from the data.

Complementary measurements of the transient response of $\mathrm{CO}_{2}$ to deeper and slower breathing were also made, where a participant carried out a series of four deep inhalations and three deep exhalations, with each cycle (inhalation + exhalation) taking about 45 seconds. These measurements were made after the participant had already been wearing the singing mask for many minutes. The observed time-varying concentrations during each inhalation and each exhalation were fit to an exponential function with an offset to determine the asymptotic $\mathrm{CO}_{2}$ concentrations.

\section{Results and Discussion}

\subsection{Through-mask filtration efficiencies for exhaled particles}

Consistent with previous findings [Alsved et al., 2020] the observed particle emission rates using the APS for singing $\left(\left\langle\dot{N}_{p, \text { sing }}\right\rangle=11.9 \mathrm{p} / \mathrm{s}\right)$ exceeded those for talking $\left(\left\langle\dot{N}_{p, \text { talk }}\right\rangle=3.3 \mathrm{p} / \mathrm{s}\right.$ by about a factor of three when no mask was used (Figure S5), similar also to mass-based results [Gregson et al., 2021]. The particle size distributions generally resembled each other for speaking and singing, although singing led to a slight enhancement in the number of particles between about $1 \mu \mathrm{m}$ and $5 \mu \mathrm{m}$ (Figure S6). Singing and talking differ somewhat in their physiological underpinnings. Singing and 'projecting' the voice typically involve a more rapid closing phase of the vocal folds, resulting in more high-frequency energy in the voice-source spectrum and a louder output sound [Lindblom and Sundberg, 2007]. The higher vocal fold velocities, and the higher degree of vocal fold tension required to produce these higher velocities, could both influence the particle sizes and numbers produced by singers. Singing also requires different valving strategies at the larynx to keep subglottal pressure (and loudness) constant throughout a single 'phrase,' which could also result in different particle size distributions between speaking and singing [Rubin 
et al., 1967]. Regardless of the physiological origin, this small variability in the inherent particle size distribution between singing and speaking will have little influence on the efficiency with which the mask reduces emission of particles from expiration to the surrounding environment.

Representative aerosol emission data from one individual singing Ludwig van Beethoven's Ode to Joy with no mask or while wearing various facemasks, including the singing mask, demonstrate that the efficiency of expiratory particle filtration for airflow passing through the mask material varies with mask type (Figure 3). Notably, this through-mask efficiency for respiratory particles averaged across all particle sizes $\left(\eta_{F}\right)$ for the singing mask is as high as for an N95 and higher than that for two different types of surgical masks, as well as for various cloth masks including two made of the same cloth as the singing mask. Considering multiple participants, the $\eta_{F}$ while speaking the Rainbow Passage [Fairbanks, 1960], singing Ode to Joy, and singing other user-selected songs was very high for the singing mask, with the average $\eta_{F}>99.5 \%$ observed for all activities and the lowest $\eta_{F}$ for a single individual of only $96.5 \%$ (Figure $4 a$ ). This high throughmask efficiency exceeds that observed for medical-grade surgical masks (Figure 4b) and for KN95 masks during speaking maneuvers [Asadi et al., 2020a] but is similar to that for an N95 as noted above (Figure 3). With respect to sound, the mask effectively acts as a low-pass filter (Figure S7), consistent with previous findings on speech and mask wearing [Saeidi et al., 2016]. The good aerosol filtering differs notably from a homemade cloth masks made from t-shirts- these had particle emission rates higher than observed without the mask owing to shedding of mask fibers, which may act as aerosolized fomites [Asadi et al., 2020a]. The high filtering efficiency of the singing mask occurs despite considerable shedding when participants intentionally rubbed the mask against itself, (such as might occur with opening/closing the bottom for potential water sipping), with a shedding rate greater than for the homemade cloth masks [Asadi et al., 2020a] (geometric mean $=20 \mathrm{p} / \mathrm{s}$ for the singing mask vs. $1 \mathrm{p} / \mathrm{s}$ for the homemade mask; Figure S8). Most likely, this distinct lack of shedding during use for the singing mask results from separation of the mask material and the wearers face, thus eliminating contact abrasion of the mask material.

The through-mask filtration efficiency for the singing mask, and for a modified singing mask lacking the opening for drinking, exceeded that for two-layer and three-layer masks made of the same cloth but having a pleated surgical-mask style (Figure S9). The pleated masks reduced the through-mask particle emission while singing Ode to Joy by a substantial, but comparably small, 
factor of $\sim 14$ while the singing masks reduced through-mask emission by >500 fold Figure 3). This observation suggests that an increase in mask-mouth separation distance for the singing mask engenders an increase in the $\eta_{F}$ and potentially helps to explain the high filtration efficiency for the cotton fabric singing mask.

\subsection{Influence of mask-face separation distance on filtration efficiency}

To assess the impact of this separation distance on $\eta_{F}$ in a controlled manner, we performed systematic experiments using a non-shedding fibrous filter having moderate efficiency (Figure 2a). Notably, the observed $\eta_{F}$ increased with the filter-mouth separation distance (Figure 2b), consistent with the difference between the two- and three-layer masks and the singing mask. This observation further supports the idea that the larger than typical mask-mouth distance of the singing mask importantly contributes to its high through-mask filtration efficiency.

The observed dependence of $\eta_{F}$ on distance $(x)$ is well described by the empirical relationship $\eta_{F}=1-\exp \left(-K^{*} \cdot x^{p}\right)$, with $p=0.45(\mathrm{~b})$. Theory indicates the overall filtration efficiency for a fibrous filter varies as $\eta_{F}=1-\exp \left(-K \cdot \eta_{S F}\right)$, where the $\eta_{S F}$ is the single-fiber filtration efficiency accounting for all processes (diffusion, interception, impaction) and $K$ is a constant that depends on the filter [Flagan and Seinfeld, 1988]. The $\eta_{S F}$ for the different processes vary with the air velocity through the filter, with $\eta_{S F, \text { diff }}$ decreasing but $\eta_{S F, \text { int+imp }}$ increasing with increasing velocity. As the expiratory jet expands away from the singer the velocity decreases and the jet will impinge on a greater area of the filter material [Abkarian et al., 2020]. Thus, we expect that $\eta_{F}$ should vary with the filter-mouth separation in a manner that depends on whether diffusion or impaction dominates the particle loss process. The observations are fit equally well as the empirical equation using an expression in which the $\eta_{S F}$ derive from the theoretical relationships between velocity and $\eta_{S F, \text { diff }}$ and $\eta_{S F, i n t+i m p}$, but where the relationship between particle size and diffusivity or impaction efficiency is not explicitly considered (see Appendix A). Further, the sizedependent through-filter efficiencies $\left(\eta_{F, D_{p}}\right)$ vary with separation distance and differ by size regime (Figure 2c,d). Smaller particles correspond to smaller $\eta_{F, D_{p}}$ while exhibiting a stronger distance dependence. For particles $>2$ microns the $\eta_{F, D_{p}}$ exhibit minimal dependence on the filtermouth separation distance. Both the increase in $\eta_{F}$ with distance and the stronger distance dependence of $\eta_{F, D_{p}}$ for the smaller particles are somewhat surprising given that diffusion-driven 
losses are typically considered minor for particles $>0.5$ microns and impaction-driven losses should decrease as velocity decreases [Flagan and Seinfeld, 1988].

Our observations differ from those of Drewnick et al. [2021], who measured size-specific filtration efficiencies as a function of face velocity for various cloth masks using a standard test procedure wherein the filter is secured in a sample holder and the airflow rate is varied to change the velocity. Specifically, for 2.5 micron particles they found that $\eta_{F, D_{p}}$ increased with velocity and for 0.5 micron particles there was little dependence of $\eta_{F, D_{p}}$ on velocity. The difference in methodologies used provides a potential explanation for this difference in behavior. In our case, the air velocity and the jet-impinged area co-vary; velocity decreases with the filter-mouth separation owing to expansion of the expiratory jet and an increase in the filter area impacted by the expiratory air. This could potentially lead to differences in how the filter material structurally responds to changes in velocity compared to the standard test methods, which could in turn impact filtration efficiency. Perhaps greater deformation of the filter material at close mask-face separation distances, corresponding to high velocity with small impacted area, occurs and reduces impaction-driven losses. We note that our methodology better reflects the physical situation that occurs with actual mask wearing compared to the standard method. While we cannot pinpoint a specific reason for the observed distance dependence with certainty, our observations indicate that the increased mask-face distance for the singing mask likely contributes to the particularly high through-mask filtration efficiency.

\subsection{Impact of mask leakage on the overall filtration efficiency for exhaled particles}

The overall mask performance additionally depends on the extent to which particles escape from the edges of the mask; the above discussion only addresses air that passes through the mask material. Any leakage that results from imperfect sealing between the mask and wearers' face reduces all overall mask efficiencies [Cappa et al., 2021; Grinshpun et al., 2009; Oberg and Brosseau, 2008]. For high-quality filtering materials, the extent of leakage is the primary determinant of overall mask efficiency $(\eta)$ [Grinshpun et al., 2009]. Following from our previous study on surgical mask leakage during speaking [Cappa et al., 2021], we determined the emission rates of particles from the top, bottom, and side quadrants of the singing mask while participants sang Ode to Joy (Figure S1). We note that negligible particle emissions from the opening for drinking (see methods) were observed after it was sealed closed. The observed particle emission 
rates from these quadrants exceeded those for the forward, through-the-mask material direction (Figure 5a). Nonetheless, wearing a mask substantially decreases the observed particle emission rates from the no-mask condition in all directions.

\section{Figure 5}

The greatest between-participant variability in the absolute emission rate occurs for sampling from the mask top quadrant, near the nose (geometric standard deviation $=0.85$ ). This suggests a greater sensitivity of emissions in this direction to how well the mask seals around the nose, which can result from individuals wearing the masks with different tightness. We confirmed this by having one participant sing Ode to Joy while securing the mask with (i) only the ear loops snug, but not tight, (ii) only the ear loops tight, and (iii) the ear loops tight plus the two head straps (Figure S10). With the snug ear loops-only, the observed particle emission rate from the mask top decreased by only a factor of three over no mask. However, with the tight ear loops-only the particle emission rate from the top decreased by a factor of 38 over no mask and with the tight ear loops and the head straps by a factor of 80 over no mask.

Sampling from the bottom quadrant yielded the largest with-mask particle emission rates. While this could indicate the greatest leakage flow in this quadrant, the associated particle size distribution differs notably from those observed in the other quadrants (Figure S11). In particular, a large-diameter mode between $~ 3-10$ microns was observed, which strongly indicates a nonexpiratory source. Owing to the extended volume of the singing mask, most participants could not avoid having the mask rub against the sampling funnel while sampling from the bottom quadrant. This strongly implicates shed fibers as a contributing particle source; consequently, the particle emission rate from the bottom quadrant provides an upper limit to leakage of expired particles.

We estimate the overall mask efficiency for reduction of expiratory aerosol emission for each individual while singing by combining the observed particle emission rates without a mask to emission from the various quadrants plus through-mask using the constrained Monte Carlo approach described in the Methods to account for splitting of the flow in the various directions (Figure 5b). The median is $98 \%$ and the mean $97 \pm 3 \%$. If the measurements from the participant who repeated the activities are combined into a single value the median is $97 \%$ and the mean $96 \%$ $\pm 4 \%$. Overall mask efficiencies of greater than $93 \%$ were determined for all but two participants wearing the singing mask. One of these individuals $(\eta=88 \%)$ had the highest normalized emission 
rate for the top quadrant, indicating that leakage around the nose contributed to this low, but still very good, efficiency. The other individual $(\eta=91 \%)$ produced a somewhat high normalized emission rate from the mask top and the second highest from the mask bottom, suggesting that both leakage and, likely, shed mask fibers contributed. (We note that these values were determined when using the median values in place of the directly observed values for the few cases for which particle shedding likely had a major impact; see Methods. When the directly observed values are used the average $\eta=96 \pm 5 \% \%$, the median is still $98 \%$, and all but three of the individual $\eta$ were $>90 \%$; see Figure S12). Regardless, the observed high overall efficiencies indicate that with proper fit, in particular ensuring a good seal around the nose, the singing mask provides substantial reduction of expiratory particle emission even while singing. Also, no visible spittle (i.e., droplets much larger than those measured here) was observed with wearing the singing mask, suggesting efficient elimination of very large droplets.

The above high filtration efficiencies correspond to particles $>0.5$ microns in diameter, as characterized by the APS. Comparison between the CPC and APS measured particle concentrations indicates that the APS characterizes $30 \%$ of the total particles on average, meaning that $70 \%$ of the particles are smaller than $\sim 0.5$ microns (Figure S13a). Using the CPC measurements we find the average $\eta=90 \pm 7 \% \%$ and the median $\eta=92 \%$, somewhat lower compared to the APS but still very high (Figure S13b). This difference between the APS (particles $>0.5$ microns) and CPC (all particles) likely results from the fact that material filtration efficiencies typically exhibit a minimum at diameters between $\sim 0.1$ microns and 0.5 microns, dependent on material [Pan et al., 2021]. The probability that a given respiratory particle might contain an infectious virion decreases as particle size decreases such that very few particles $<0.5$ microns are expected to contain an infectious virion [Anand and Mayya, 2020]. Therefore, the APS measurements likely provide the most appropriate characterization of the overall filtration efficiency in the context of respiratory diseases.

The overall efficiency determined here for the singing mask greatly exceeds that found for surgical masks during speaking [Cappa et al., 2021], and further there is substantially less individual variation between participants (Figure 5b,c). The singing mask design provides a tighter fit that reduces, but does not eliminate, variability in the fit quality. This, together with the greater efficiency for air passing through the mask, leads to the overall improved performance compared to a surgical mask. Notably, the overall efficiency of the singing mask including leakage exceeds 
the efficiency of the surgical mask even if we assume zero leakage flow. While some tools exist to help improve sealing of cloth and surgical masks [Clapp et al., 2020; Rothamer et al., 2021] their use during singing is infeasible. Similarly, so-called double masking has been suggested, including by the U.S. Centers for Disease Control [Brooks et al., 2021], as a way to improve both filtering ability and fit, and thus overall efficiency [Gandhi and Marr, 2021], but this too would not be appropriate for singing. Even so, outside of singing contexts (e.g., public adoption), double masking does not ensure one obtains a good seal and thus the actual benefit is difficult to knowespecially given the wide range of cloth masks available. Also, while qualitative, the participants generally indicated that greater breathing comfort with the singing mask compared to the mask(s) each individual typically wore, most likely owing to the increased mask-face separation. We speculate that the more open design of the singing mask could increase mask wearing compliance.

\subsection{Mask filtration efficiency towards ambient particles}

While mask wearing plays an important role in reducing emission of expiratory particles, masks can also reduce inhaled particles concentrations. To address this, we measured for one participant the time-varying concentration of particles inside the singing mask during breathing (Figure 6). When leaving the mask neck seal intentionally somewhat loose the peak particle concentrations inside the mask upon inhalation approximately equaled the room air aerosol concentration. Upon exhalation the concentration fell to very low values, indicative of the lower aerosol concentration in exhaled breath compared to most ambient environments and ambient particle deposition in the respiratory system. However, when the mask was worn as designed, with the neck seal tight, the inside-mask concentration upon inhalation rose only to about $6 \%$ of the room air concentration, indicative of an effective efficiency of $94 \%$ for inhalation, similar to that found for expiratory particle emission. Mask performance is sometimes characterized by the fit factor, which in this case is just the ratio between the room air concentration and the concentration measured in the mask, or $\sim 16.7$ [Lindsley et al., 2021]. Distinct from expiratory aerosols, the room air aerosols were primarily smaller than 0.5 microns, with only $\sim 10 \%$ of the particles larger. Filtration efficiency typically increases with particle size above this range [Cappa et al., 2021; Drewnick et al., 2021] and thus we expect the efficiency of the singing mask towards expiratory aerosols emitted by others will be even greater than found here. Similar to exhalation, the singing mask geometry likely contributes to the high filtration efficiency towards ambient particles during 
inhalation. The greater mask-face separation will lead to air being drawn through nearly the entirety of the mask surface area, with a consequently lower face velocity and increased filtration efficiency compared to conventional designs. Overall, this demonstrates that wearing of the singing mask provides major benefits to both the wearer and others.

\subsection{Deadspace microclimate}

Concentrations of $\mathrm{CO}_{2}$ in the deadspace of filtering facepiece respirators, such as N95 respirators, are substantially elevated above ambient concentrations ( $\sim 20 \mathrm{ppm})$, with consequent concerns over $\mathrm{CO}_{2}$ rebreathing [Smith et al., 2013]. For example, Rhee et al. [2021], found the steady state $\left[\mathrm{CO}_{2}\right]=26,000 \mathrm{ppm}_{\mathrm{v}}$ inside the respirator deadspace for participants wearing KN95 respirators. This high concentration results from the high $\left[\mathrm{CO}_{2}\right]$ in exhaled breath of $\sim 5 \%$ by volume, or 50,000 $\mathrm{ppm}_{\mathrm{v}}$. The deadspace of the singing mask is about 3-5 times larger than most facepiece respirators. Thus, there is potential concern over $\mathrm{CO}_{2}$ buildup in the singing mask. Correspondingly, as the $\mathrm{CO}_{2}$ in exhaled air derives from the inhaled $\mathrm{O}_{2}$, limited exchange of mask air with ambient air could result in $\mathrm{O}_{2}$ depletion. Our measurements show the steady state $\left[\mathrm{CO}_{2}\right]$ $\sim 19,000 \mathrm{ppm}_{\mathrm{v}}$ during speaking and singing, lower than the steady state observed by Rhee et al. [2021] for an N95 respirator and lower than the steady state measured for this same participant wearing a KN95 respirator $\left(\sim 25,000 \mathrm{ppm}_{\mathrm{v}}\right)$ (Figure $\left.7 \mathrm{a}\right)$. The steady state $\left[\mathrm{O}_{2}\right]$ measured in the singing mask averaged $18.5 \%$ by volume during speaking of the Rainbow Passage and 18.0\% during longer wearing $\left(30 \mathrm{~min}\right.$ ) by one participant (Figure $7 \mathrm{~b}$ ). While lower than the $\left[\mathrm{O}_{2}\right]$ in ambient air (20.8\%) this concentration exceeds that in exhaled breath $(\sim 16 \%)$. In terms of oxygen availability, this decrease in $\left[\mathrm{O}_{2}\right]$ corresponds to an equivalent change in altitude from sea level to about $1 \mathrm{~km}$ and is consistent with respirator use having no influence on oxygen saturation level [Kim et al., 2013]. If there were limited mask-ambient air exchange the $\left[\mathrm{CO}_{2}\right]$ should build up over time while the $\left[\mathrm{O}_{2}\right]$ should continually deplete. That this did not occur, even over 30 minutes of wearing, demonstrates substantial exchange occurs.

Our complementary transient measurements during breathing show that the deadspace $\left[\mathrm{CO}_{2}\right]$ asymptotically approaches $\sim 36,000 \mathrm{ppm}_{\mathrm{v}}$ during exhalation and $\sim 6,500 \mathrm{ppm}_{\mathrm{v}}$ during inhalation (Figure S14). These observations demonstrate that there is exchange between the deadspace and ambient environment during both exhalation and inhalation, but with some residual in the deadspace that is not fully cleared out. If exchange did not occur we would expect that the 
deadspace $\left[\mathrm{CO}_{2}\right]$ would asymptote to the value in exhaled breath during exhalation and if there were no residual in the deadspace we would expect $\mathrm{CO}_{2}$ to asymptote to ambient values during inhalation.

To understand the steady state and transient observations we have built a simple model of ambient-mask air exchange, inhalation and exhalation, and $\mathrm{O}_{2} \rightarrow \mathrm{CO}_{2}$ conversion upon inhalation during breathing (see Supplemental Materials). The model indicates that inhaled air comprises about $85 \%$ of ambient air and $15 \%$ of deadspace air, leading to an average $\left[\mathrm{CO}_{2}\right]$ in inhaled air of $\sim 6,000 \mathrm{ppm}_{\mathrm{v}}$. This concentration is slightly higher than the $8 \mathrm{~h}$ time-weighted average occupational exposure limit for $\mathrm{CO}_{2}$ of 5,000 $\mathrm{ppm}_{\mathrm{v}}$ and well below the short-term exposure limit of 30,000 $\mathrm{ppm}_{\mathrm{v}}$ [OSHA, 2021]. During exhalation, about $15 \%$ of the air is residual deadspace air, $25 \%$ of the air is exchanged ambient air, and $60 \%$ is exhaled air. The model consideration confirms that substantial mask-ambient air exchange occurs. Overall, given these calculations and the general similarity of the steady state $\mathrm{CO}_{2}$ for the singing mask and for N95 respirators we can expect that continuous wearing (for hours) may lead to slightly elevated transcutaneous $\mathrm{CO}_{2}$ levels of wearers, but at levels that are not clinically important [Scheid et al., 2020].

The relative humidity in the singing mask deadspace averaged $\sim 65 \%$ for both speaking and singing, and the temperature averaged $\sim 31^{\circ} \mathrm{C}$ (Figure 7c,d). The temperature in the singing mask was similar to that measured in the KN95 respirator $\left(\sim 31^{\circ} \mathrm{C}\right)$ whereas the relative humidity was much lower than that in the KN95 respirator ( 90\%). Our observations for the KN95 respirator are consistent with previous measurements for N95 respirators [Roberge et al., 2012]. The higher relative humidity in the KN95 mask and in N95 masks generally could result from greater water retention by the KN95 material compared to the singing mask material.

Anecdotally, a few participants noted that the air felt somewhat stale while singing with mask wearing, indicating some level of discomfort compared with no mask wearing. The participant who performed numerous repeats indicated that comfort increased with repeated use on multiple occasions, suggesting that there may be some aspect of acclimatization. That same participant also wore the singing mask for $>1 \mathrm{~h}$ while working quietly and did not note any particular physical discomfort beyond feeling a slightly elevated skin temperature. Further research would be necessary to quantify mask wearer's subjective or physiological responses beyond these anecdotal observations. 


\section{Summary}

In summary, the overall filtering efficiency of the cloth singing mask when fit properly compares favorably to that expected for N95 respirators both for filtration of exhaled respiratory particles and inhalation of ambient particles. The higher efficiency results, in part, from the substantial mask-face distance compared to typical mask designs. Sufficient exchange between air in the mask deadspace and the ambient environment constrains $\mathrm{CO}_{2}$ buildup below that observed in N95 respirators and prevents physiologically important $\mathrm{O}_{2}$ depletion. Additionally, the relative humidity in the singing mask is lower than that in N95 masks ( 65\% compares to $90 \%$, respectively). As the materials comprising the singing mask and N95s differ, the singing mask, or similarly designed masks, could provide a viable alternative to N95s outside of healthcare or other specialized occupational settings for situations when greater mask efficiency is necessary or desired. For singers and other performers, in particular, the singing mask, when fit properly, has sufficiently high efficiency - maintained throughout the act of singing - that its adoption could facilitate in-person rehearsals with multiple people as long as other best practices (e.g., good ventilation) are also adopted.

\section{Acknowledgements}

The authors greatly appreciate the participation of the various professional and amateur singers who volunteered their time. We thank Helen Dahlke at UC Davis for use of the $\mathrm{CO}_{2}$ and $\mathrm{O}_{2}$ sensors. Author contributions: C. D. C. led the measurements and analysis. S. A. R. and A. A-K. of the San Francisco Opera (SFO) designed the mask. SFO constructed the masks and SFO and C. D. C provided design suggestions. W. D. R. contributed to experimental design and interpretation. E. L. facilitated the $\mathrm{CO}_{2}$ and $\mathrm{O}_{2}$ measurements. A. S. W., S. B., and N. M. B. contributed to interpretation. C. D. C, and S. A. R. led the manuscript writing. All authors reviewed and revised the manuscript for accuracy and intellectual content. Competing interests: S. A. R. and A. A-K., have filed a patent application for the singing mask. All other authors declare no competing interests. Data and materials availability: All data needed to evaluate the conclusions in the paper are present in the paper and/or the supplementary materials. Additional data and files related to this paper will be made publicly available at doi:10.25338/B8GD1B and are available for review at https://bit.ly/3qUJpiy. 


\section{Funding}

N/A

\section{Appendix A}

For an expiratory jet, the air velocity, $u$, decreases with distance as $1 /(\alpha \cdot x)$, where $\alpha$ is the divergence angle $\left(\sim 20^{\circ}\right)$ and $x$ is distance [Abkarian et al., 2020]. Overall filtration efficiencies $\left(\eta_{f}\right)$ for fibrous filters vary as:

$\eta_{f}=1-\exp \left(-K \cdot \eta_{S F}\right)$

where $\eta_{S F}$ is the single-fiber filtration efficiency accounting for losses by diffusion, interception, and impaction and $K$ is a constant dependent on the filter packing density, thickness, and fiber size [Flagan and Seinfeld, 1988]. For diffusion-driven losses,

$\eta_{S F, \text { diff }} \propto P e^{-\frac{2}{3}} \propto u^{-\frac{2}{3}}$

and where $P e$ is the Peclet number, which is proportional to velocity $(u)$ through the filter [Flagan and Seinfeld, 1988]. Loss via interception and impaction has a more complex relationship with conditions, but can be approximated as

$\eta_{S F, i n t+i m p} \sim 10^{\wedge}\left[y_{0}+\frac{a b s\left(y_{0}\right)}{1+\exp \left(\frac{\left(\log \left(S t k_{0}\right)-\log (S t k)\right)}{r}\right)}\right]$,

where $S t k$ is the Stokes number, which is proportional to velocity and the other terms are constants that depend on the fiber size and the particle diameter [Flagan and Seinfeld, 1988]. The overall single-fiber efficiency is then:

$\eta_{S F}=\eta_{S F, \text { diff }}+\eta_{S F, \text { int }+ \text { imp }}-\eta_{S F, \text { diff }} \cdot \eta_{S F, \text { int }+ \text { imp }}$

Rewriting Eqn. S2 and S3 to depend on the mask-face separation distance, $x$, rather than velocity, we have:

$\eta_{S F, \text { diff }}=A \cdot x^{\frac{2}{3}}$

and

$\eta_{S F, \text { int }+ \text { imp }} \sim 10^{\wedge}\left[y_{0}+\frac{a b s\left(y_{0}\right)}{1+\exp \left(\frac{\left(-\log \left(x_{0}\right)-\log (x)\right)}{r}\right)}\right]$. 
Substitution of Eqn. S5 and S6 into Eqn. S4, and the resulting expression into Eqn. S1 yields an expression for the overall filtration efficiency that depends on the mask-face separation distance, and which can be fit to observations.

\section{References}

Abkarian, M., S. Mendez, N. Xue, F. Yang, and H. A. Stone (2020), Speech can produce jet-like transport relevant to asymptomatic spreading of virus, Proceedings of the National Academy of Sciences, 117(41), 25237-25245, doi:10.1073/pnas.2012156117.

Alsved, M., A. Matamis, R. Bohlin, M. Richter, P. E. Bengtsson, C. J. Fraenkel, P. Medstrand, and J. Löndahl (2020), Exhaled respiratory particles during singing and talking, Aerosol Science and Technology, 54(11), 1245-1248, doi:10.1080/02786826.2020.1812502.

Anand, S., and Y. S. Mayya (2020), Size distribution of virus laden droplets from expiratory ejecta of infected subjects, Scientific Reports, 10(1), 21174, doi:10.1038/s41598-020-78110-x.

Asadi, S., C. D. Cappa, S. Barreda, A. S. Wexler, N. M. Bouvier, and W. D. Ristenpart (2020a), Efficacy of masks and face coverings in controlling aerosol particle emission from expiratory activities, Scientific Reports, 10, 15665, doi:10.1038/s41598-020-72798-7.

Asadi, S., N. Gaaloul ben Hnia, R. S. Barre, A. S. Wexler, W. D. Ristenpart, and N. M. Bouvier (2020b), Influenza A virus is transmissible via aerosolized fomites, Nature Communications, 11(1), 4062, doi:10.1038/s41467-020-17888-w.

Asadi, S., A. S. Wexler, C. D. Cappa, S. Barreda, N. M. Bouvier, and W. D. Ristenpart (2019), Aerosol emission and superemission during human speech increase with voice loudness, Scientific Reports, 9(1), 2348, doi:10.1038/s41598-019-38808-z.

Asadi, S., A. S. Wexler, C. D. Cappa, S. Barreda, N. M. Bouvier, and W. D. Ristenpart (2020c), Effect of voicing and articulation manner on aerosol particle emission during human speech, PLoS One, 15(1), e0227699, doi:10.1371/journal.pone.0227699.

ASTM International (2017), ASTM Standard F2299, in Standard Test Method for Determining the Initial Efficiency of Materials Used in Medical Face Masks to Penetration by Particulates Using Latex Spheres, edited, West Conshohocken, PA.

Bagheri, M. H., I. Khalaji, A. Azizi, R. T. Loibl, N. Basualdo, S. Manzo, M. L. Gorrepati, S. Mehendale, C. Mohr, and S. N. Schiffres (2021), Filtration efficiency, breathability, and reusability of improvised materials for face masks, Aerosol Science and Technology, 1-15, doi:10.1080/02786826.2021.1898537.

Binazzi, B., B. Lanini, R. Bianchi, I. Romagnoli, M. Nerini, F. Gigliotti, R. Duranti, J. MilicEmili, and G. Scano (2006), Breathing pattern and kinematics in normal subjects during speech, singing and loud whispering, Acta Physiologica, 186(3), 233-246, doi:https://doi.org/10.1111/j.1748-1716.2006.01529.x.

Brooks, J. T., D. H. Beezhold, J. D. Noti, J. P. Coyle, R. C. Derk, F. M. Blachere, and W. G. Lindsley (2021), Maximizing Fit for Cloth and Medical Procedure Masks to Improve Performance and Reduce SARS-CoV-2 Transmission and Exposure, MMWR Morb Mortal Wkly Rep, doi:http://dx.doi.org/10.15585/mmwr.mm7007e1. 
Brooks, J. T., and J. C. Butler (2021), Effectiveness of Mask Wearing to Control Community Spread of SARS-CoV-2, JAMA, doi:10.1001/jama.2021.1505.

Cappa, C. D., S. Asadi, S. Barreda, A. S. Wexler, N. M. Bouvier, and W. D. Ristenpart (2021), Expiratory aerosol particle escape from surgical masks due to imperfect sealing, Scientific Reports, 11, 12110, doi:10.1038/s41598-021-91487-7.

Chand, M., et al. (2021), Investigation of novel SARS-CoV-2 variant, Variant of Concern 202012/01; Technical briefing 2,

https://assets.publishing.service.gov.uk/government/uploads/system/uploads/attachment_data/file 1948152/Technical_Briefing_VOC202012-2_Briefing_2_FINAL.pdf,

Clapp, P. W., E. E. Sickbert-Bennett, J. M. Samet, J. Berntsen, K. L. Zeman, D. J. Anderson, D. J. Weber, W. D. Bennett, U. C. f. D. Control, and P. E. Program (2020), Evaluation of Cloth Masks and Modified Procedure Masks as Personal Protective Equipment for the Public During the COVID-19 Pandemic, JAMA Internal Medicine, doi:10.1001/jamainternmed.2020.8168.

Clark, R. P., and S. G. Shirley (1973), Identification of Skin in Airborne Particulate Matter, Nature, 246(5427), 39-40, doi:10.1038/246039a0.

Dai, T., G. Bai, and G. F. Anderson (2020), PPE Supply Chain Needs Data Transparency and Stress Testing, Journal of General Internal Medicine, 35(9), 2748-2749, doi:10.1007/s11606020-05987-9.

Drewnick, F., J. Pikmann, F. Fachinger, L. Moormann, F. Sprang, and S. Borrmann (2021), Aerosol filtration efficiency of household materials for homemade face masks: Influence of material properties, particle size, particle electrical charge, face velocity, and leaks, Aerosol Science and Technology, 55(1), 63-79, doi:10.1080/02786826.2020.1817846.

Fairbanks, G. (1960), Voice and Articulation Drillbook, 2nd ed., 234 pp., Harper and Row, New York.

Flagan, R. C., and J. H. Seinfeld (1988), Removal of particles from gas streams, in

Fundamentals of air pollution engineering, edited, pp. 391-478, Prentice-Hall, Englewood Cliffs, N.J.

Gandhi, M., and L. C. Marr (2021), Uniting Infectious Disease and Physical Science Principles on the Importance of Face Masks for COVID-19, Med, 2(1), 29-32, doi:10.1016/j.medj.2020.12.008.

Gregson, F. K. A., et al. (2021), Comparing aerosol concentrations and particle size distributions generated by singing, speaking and breathing, Aerosol Science and Technology, 1-15, doi:10.1080/02786826.2021.1883544.

Grinshpun, S. A., H. Haruta, R. M. Eninger, T. Reponen, R. T. McKay, and S.-A. Lee (2009), Performance of an N95 Filtering Facepiece Particulate Respirator and a Surgical Mask During Human Breathing: Two Pathways for Particle Penetration, Journal of Occupational and Environmental Hygiene, 6(10), 593-603, doi:10.1080/15459620903120086.

Gupta, J. K., C.-H. Lin, and Q. Chen (2010), Characterizing exhaled airflow from breathing and talking, Indoor Air, 20(1), 31-39, doi:10.1111/j.1600-0668.2009.00623.x. 
Hallett, S., F. Toro, and J. V. Ashurst (2020), Physiology, Tidal Volume, in StatPearls [Internet], edited, p. https://www.ncbi.nlm.nih.gov/books/NBK482502/, StatPearls Publishing, Treasure Island, FL.

Holmberg, E. B., R. E. Hillman, and J. S. Perkell (1988), Glottal airflow and transglottal air pressure measurements for male and female speakers in soft, normal, and loud voice, The Journal of the Acoustical Society of America, 84(2), 511-529, doi:10.1121/1.396829.

Hospodsky, D., J. Qian, W. W. Nazaroff, N. Yamamoto, K. Bibby, H. Rismani-Yazdi, and J. Peccia (2012), Human Occupancy as a Source of Indoor Airborne Bacteria, PLoS One, 7(4), e34867, doi:10.1371/journal.pone.0034867.

Howard, J., et al. (2021), An evidence review of face masks against COVID-19, Proceedings of the National Academy of Sciences, 118(4), e2014564118, doi:10.1073/pnas.2014564118.

Jiang, J. J., R. B. Hanna, M. V. Willey, and A. Rieves (2016), The Measurement of Airflow Using Singing Helmet That Allows Free Movement of the Jaw, Journal of Voice, 30(6), 641648, doi:https://doi.org/10.1016/j.jvoice.2015.07.018.

Kim, J.-H., S. M. Benson, and R. J. Roberge (2013), Pulmonary and heart rate responses to wearing N95 filtering facepiece respirators, American Journal of Infection Control, 41(1), 24-27, doi:10.1016/j.ajic.2012.02.037.

Kwon, S.-B., J. Park, J. Jang, Y. Cho, D.-S. Park, C. Kim, G.-N. Bae, and A. Jang (2012), Study on the initial velocity distribution of exhaled air from coughing and speaking, Chemosphere, 87(11), 1260-1264, doi:10.1016/j.chemosphere.2012.01.032.

Lindblom, B., and J. Sundberg (2007), The Human Voice in Speech and Singing, in Springer Handbook of Acoustics, edited by T. Rossing, Springer, New York, NY, doi:10.1007/978-0-38730425-0_16.

Lindsley, W. G., et al. (2021), A comparison of performance metrics for cloth masks as source control devices for simulated cough and exhalation aerosols, Aerosol Science and Technology, 118, doi:10.1080/02786826.2021.1933377.

Miller, S. L., W. W. Nazaroff, J. L. Jimenez, A. Boerstra, G. Buonanno, S. J. Dancer, J. Kurnitski, L. C. Marr, L. Morawska, and C. Noakes (2021), Transmission of SARS-CoV-2 by inhalation of respiratory aerosol in the Skagit Valley Chorale superspreading event, Indoor Air, 31, 314-323, doi:10.1111/ina.12751.

Oberg, T., and L. M. Brosseau (2008), Surgical mask filter and fit performance, American Journal of Infection Control, 36(4), 276-282, doi:10.1016/j.ajic.2007.07.008.

Occupational Safety and Health Administration (2004), Fit Testing Procedures (Mandatory), in Occupational Safety and Health Standards, Personal Protective Equipment, edited.

OSHA (2021), Permissible Exposure Limits - Annotated Tables, https://www.osha.gov/annotated-pels/table-z-1, Accessed: 8 July 2021.

Pan, J., C. Harb, W. Leng, and L. C. Marr (2021), Inward and outward effectiveness of cloth masks, a surgical mask, and a face shield, Aerosol Science and Technology, 55(6), 718-733, doi:10.1080/02786826.2021.1890687. 
Rhee, M. S. M., C. D. Lindquist, M. T. Silvestrini, A. C. Chan, J. J. Y. Ong, and V. K. Sharma (2021), Carbon dioxide increases with face masks but remains below short-term NIOSH limits, BMC Infectious Diseases, 21(1), 354, doi:10.1186/s12879-021-06056-0.

Roberge, R. J., J.-H. Kim, and S. Benson (2012), N95 Filtering Facepiece Respirator Deadspace Temperature and Humidity, Journal of Occupational and Environmental Hygiene, 9(3), 166-171, doi:10.1080/15459624.2012.660428.

Rothamer, D. A., S. Sanders, D. Reindl, and T. H. Bertram (2021), Strategies to minimize SARS-CoV-2 transmission in classroom settings: Combined impacts of ventilation and mask effective filtration efficiency, medRxiv, 2020.2012.2031.20249101, doi:10.1101/2020.12.31.20249101.

Rubin, H. J., M. LeCover, and W. Vennard (1967), Vocal Intensity, Subglottic Pressure and Air Flow Relationships in Singers, Folia Phoniatrica et Logopaedica, 19(6), 393-413, doi:10.1159/000263170.

Saeidi, R., I. Huhtakallio, and P. Alku (2016), Analysis of Face Mask Effect on Speaker Recognition, paper presented at INTERSPEECH 2016, International Speech Communication Association, San Francisco.

Scheid, J. L., S. P. Lupien, G. S. Ford, and S. L. West (2020), Commentary: Physiological and Psychological Impact of Face Mask Usage during the COVID-19 Pandemic, International Journal of Environmental Research and Public Health, 17(18), 6655.

Shakya, K. M., A. Noyes, R. Kallin, and R. E. Peltier (2017), Evaluating the efficacy of cloth facemasks in reducing particulate matter exposure, Journal of Exposure Science \& Environmental Epidemiology, 27(3), 352-357, doi:10.1038/jes.2016.42.

Smith, C. L., J. L. Whitelaw, and B. Davies (2013), Carbon dioxide rebreathing in respiratory protective devices: influence of speech and work rate in full-face masks, Ergonomics, 56(5), 781-790, doi:10.1080/00140139.2013.777128.

Taylor, S., and G. J. G. Asmundson (2020), Negative attitudes about facemasks during the COVID-19 pandemic: The dual importance of perceived ineffectiveness and psychological reactance, medRxiv, 2020.2011.2017.20233585, doi:10.1101/2020.11.17.20233585.

$\mathrm{Xu}$, M., Z. Lei, and J. Yang (2015), Estimating the Dead Space Volume Between a Headform and N95 Filtering Facepiece Respirator Using Microsoft Kinect, Journal of Occupational and Environmental Hygiene, 12(8), 538-546, doi:10.1080/15459624.2015.1019078. 


\section{Figure Captions}

Figure 1. (a) Top and bottom view of the singing mask. (b) (top) Images of one participant wearing a surgical mask and wearing the singing mask. (bottom) Overlaid images of the participant with the surgical mask and with a singing mask to illustrate the increased mask-face material separation in the singing mask. The images in (b) were modified from the originals using the "glow edges" artistic effect in Microsoft PowerPoint to accentuate the mask edges and to overlay an image of the participant with no mask. Informed consent was obtained.

Figure 2. (a) Schematic of sampling setup for measuring the influence of the filter-face separation distance on the filtration efficiency of a fibrous filter. (b) Filtration efficiency measured as a function of the filter-face distance measured for one participant in triplicate where the filtration material was the outer-layer of surgical mask \#1. Fits correspond to a simple power law and a full expression that distinguishes particle loss mechanisms. (c) Measured particle size distributions for no filter (black) and for a fibrous filter (colors) with the participant speaking the Rainbow Passage at varying distances filter-face separation distances. (d) The size-dependent filtration efficiency as a function of the filter-face separation distance, for different size bins indicated with the gray vertical lines in (c).

Figure 3. (left axis) Bars showing the observed particle emission rates for one participant singing Ode to Joy with no mask, or a homemade cloth two-layer or three-layer pleated mask made of the same material as the singing mask, a modified singing mask having no bottom opening, the standard singing mask, a commercial cloth mask either without (FDPM) or with (FDPM(N95)) an N95 insert, either of two different surgical masks, or an N95 respirator (3M), as measured in the forward (through-mask) position. Note that jaw movement was substantially restricted with the non-singing masks, especially the N95 respirator. Three repeats were performed and error bars are $1 \sigma$ geometric standard deviations. The reported emission rates have not been corrected for flow. (right axis) The associated reduction efficiency for exhaled particles sampled in the forward (through mask) direction (black points).

Figure 4. (a) The through-mask (forward) particle emission rates normalized to no mask (left axis) or the corresponding reduction efficiency (right axis) observed for participants while wearing the singing mask for speaking or singing either the Ode to Joy or a song chosen by the participant. Closed colored circles in (a) indicate unique individuals while open gray circles indicate repeats by one individual. (b) Normalized particle emission rates for participants speaking while wearing a surgical mask (original data from Cappa et al. [2021]). Note that the participants in (a) differ from those in (b). Box and whisker plots show the median, $25^{\text {th }} / 75^{\text {th }}$ percentile, and $10^{\text {th }} / 90^{\text {th }}$ percentile.

Figure 5. (a) Particle emission rates normalized to no mask wearing for singing Ode to Joy for the different orientations while wearing the singing mask. (b) The overall mask efficiency while singing Ode to Joy with the singing mask, accounting for leakage flows, colored by participant (circles). Uncertainty bars on individual points indicate $1 \sigma$ determined from Monte Carlo simulations. The red square is the average across all measurements and the blue triangle the 
average after combining replicate measurements from one participant. Closed circles in (a) and (b) indicate unique individuals while open circles indicate repeats by one individual. (c) Overall efficiency for speaking with a surgical mask including leakage flows (solid points) or assuming all flow passes through the mask, i.e., zero leakage flows and perfect sealing (open points). Original data from Cappa et al. [2021], but reprocessed here for individuals. Note that the participants in $(a, b)$ differ from those in (c). Box and whisker plots show the median, $25^{\text {th }} / 75^{\text {th }}$ percentile, and $10^{\text {th }} / 90^{\text {th }}$ percentile.

Figure 6. Time-series of particle concentrations measured using a CPC for room air (solid black line), sampled inside the singing mask worn intentionally loose around the neck (blue circles), and when sampled inside the mask when worn tightly as designed (gold triangles). The right axis shows the approximate mask efficiency based on comparison with the mean room air concentration.

Figure 7. (a) $\mathrm{CO}_{2}$ and (b) $\mathrm{O}_{2}$ concentrations along with (c) relative humidity and (d) temperature measured in the deadspace of the singing mask during speaking (red) and singing (blue) and in the deadspace of a KN95 respirator during speaking (gold), compared with that for room air (black line) and in typical exhaled air (gray band). Dashed versus solid lines differentiate replicates. 


\section{Figures}
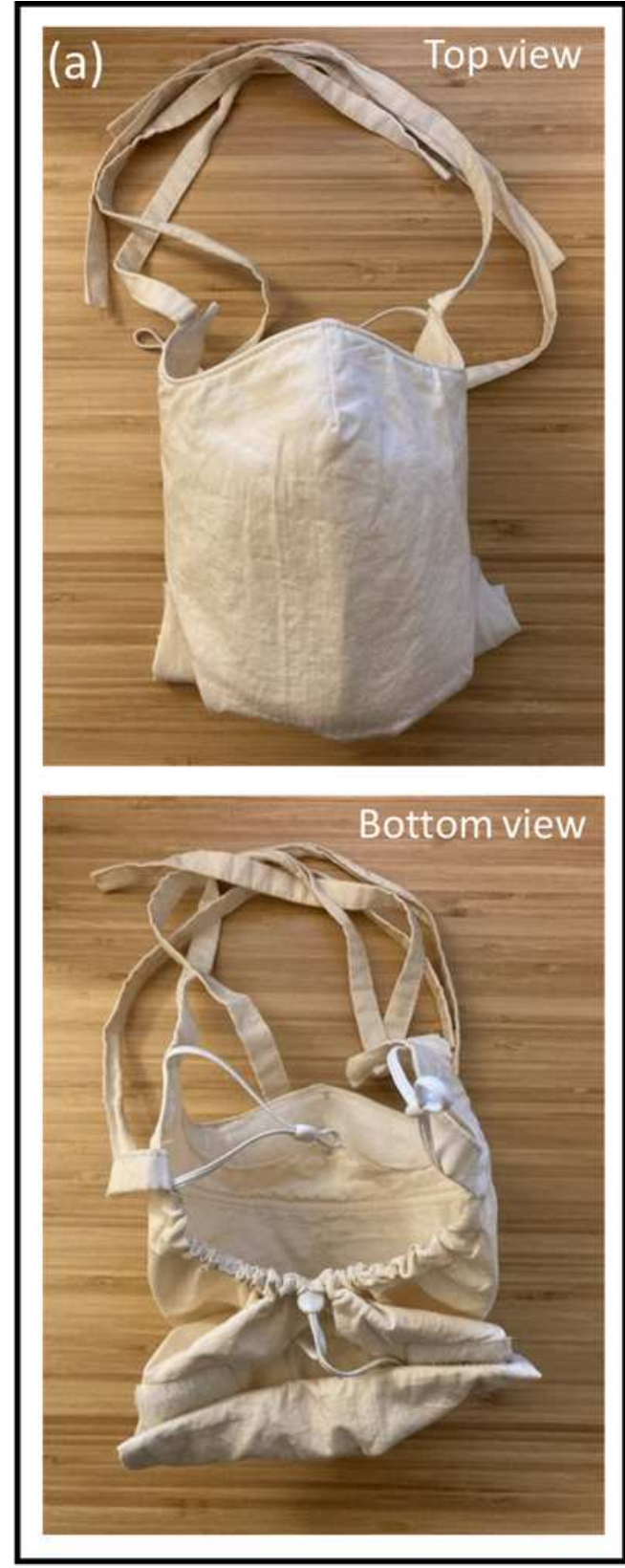
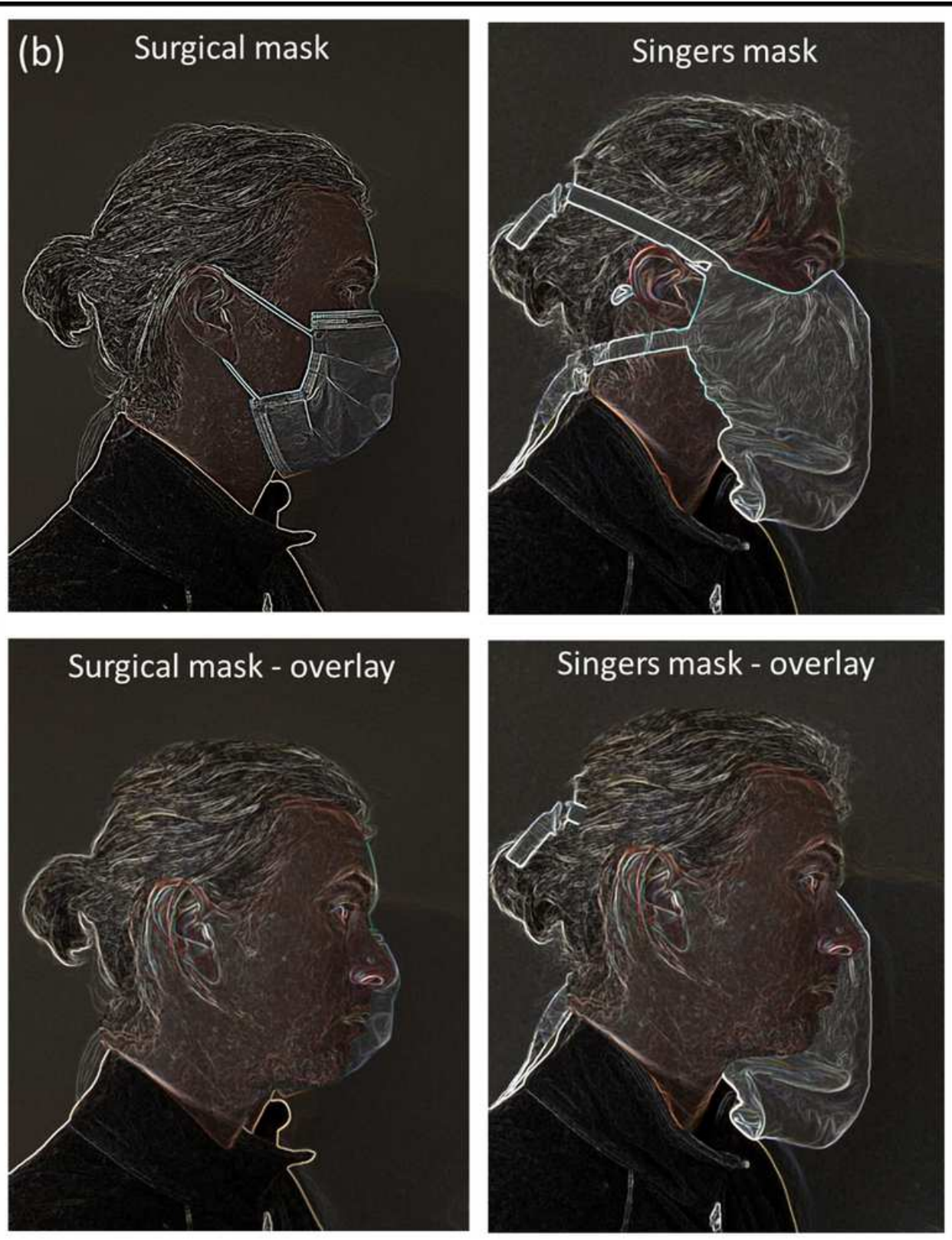

\section{Figure 1}

(a) Top and bottom view of the singing mask. (b) (top) Images of one participant wearing a surgical mask and wearing the singing mask. (bottom) Overlaid images of the participant with the surgical mask and with a singing mask to illustrate the increased mask-face material separation in the singing mask. The images in (b) were modified from the originals using the "glow edges" artistic effect in Microsoft PowerPoint to accentuate the mask edges and to overlay an image of the participant with no mask. Informed consent was obtained. 
(a)
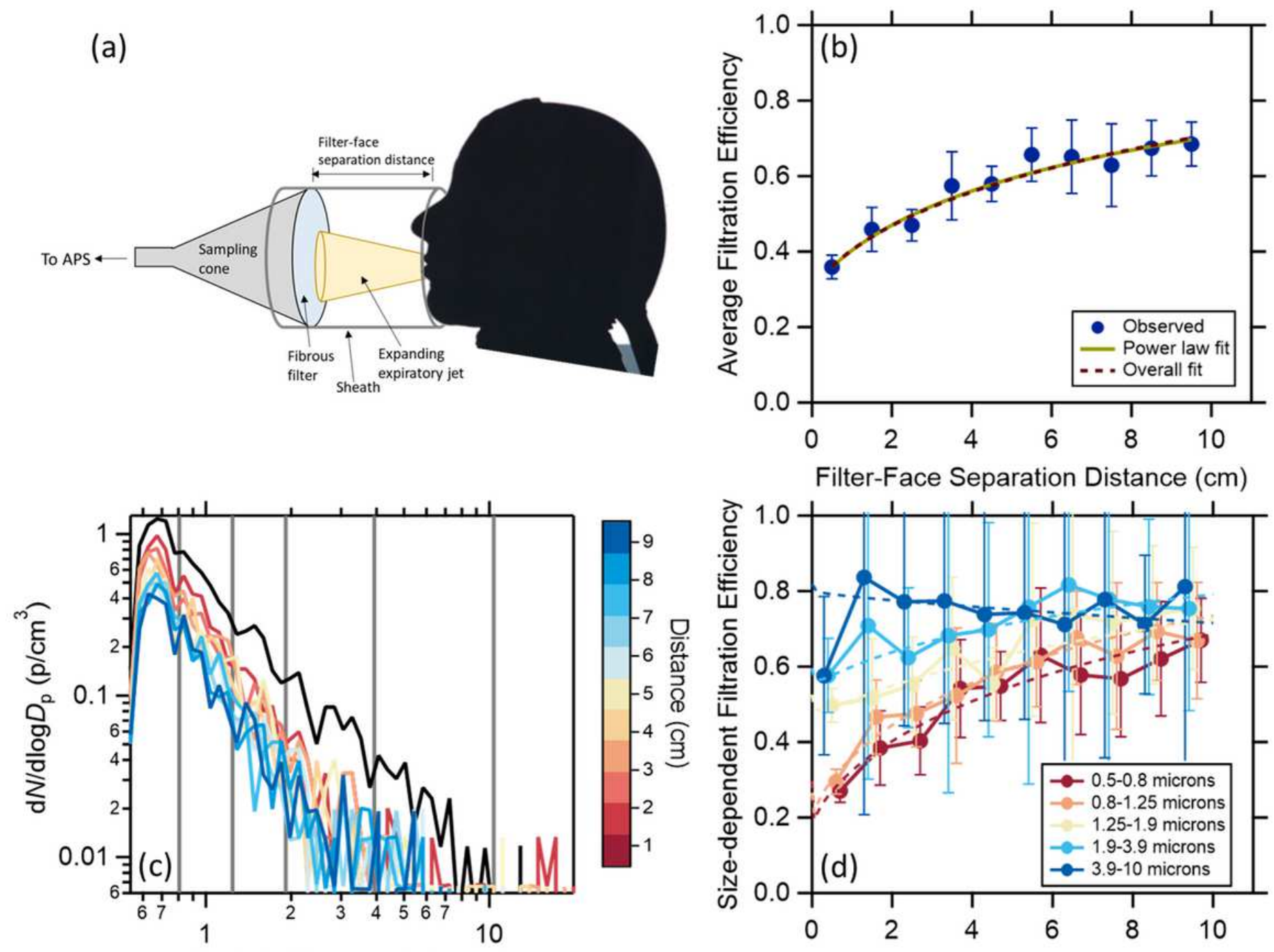

Particle Diameter (microns)

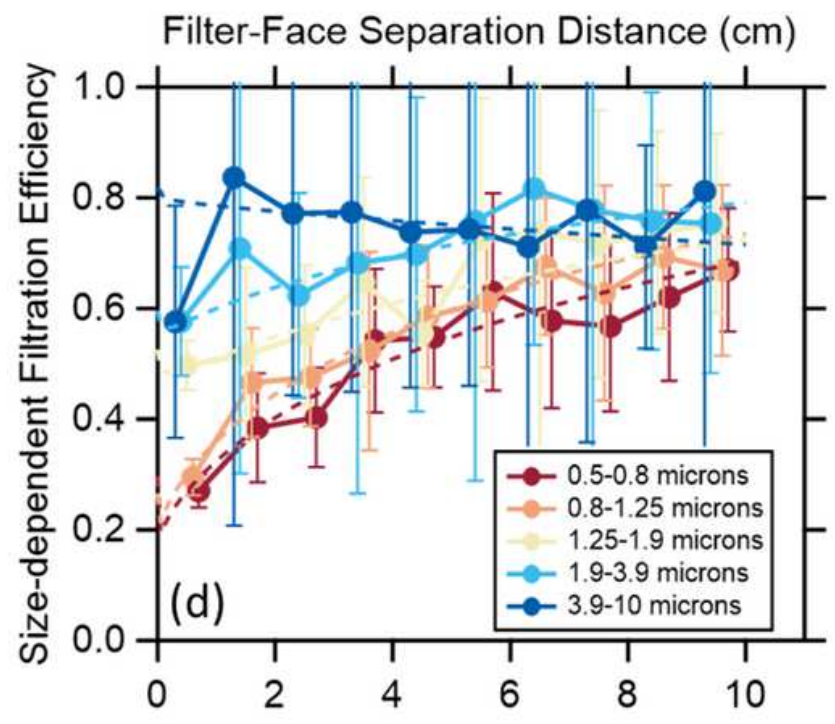

Filter-Face Separation Distance $(\mathrm{cm})$

Figure 2

(a) Schematic of sampling setup for measuring the influence of the filter-face separation distance on the filtration efficiency of a fibrous filter. (b) Filtration efficiency measured as a function of the filter-face distance measured for one participant in triplicate where the filtration material was the outer-layer of surgical mask \#1. Fits correspond to a simple power law and a full expression that distinguishes particle loss mechanisms. (c) Measured particle size distributions for no filter (black) and for a fibrous filter (colors) with the participant speaking the Rainbow Passage at varying distances filter-face separation distances. (d) The size-dependent filtration efficiency as a function of the filter-face separation distance, for different size bins indicated with the gray vertical lines in (c). 


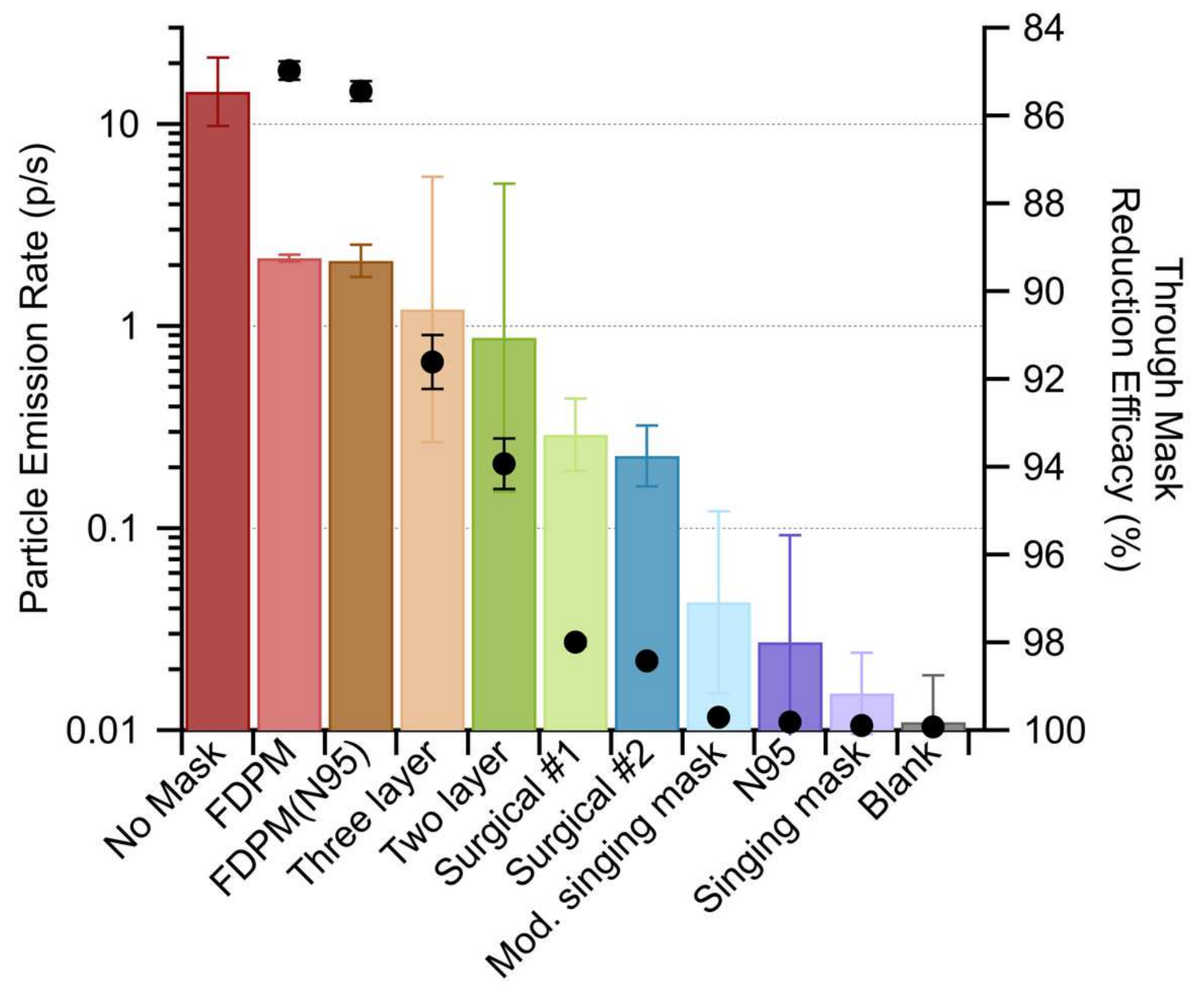

Figure 3

(left axis) Bars showing the observed particle emission rates for one participant singing Ode to Joy with no mask, or a homemade cloth two-layer or three-layer pleated mask made of the same material as the singing mask, a modified singing mask having no bottom opening, the standard singing mask, a commercial cloth mask either without (FDPM) or with (FDPM(N95)) an N95 insert, either of two different surgical masks, or an N95 respirator (3M), as measured in the forward (through-mask) position. Note that jaw movement was substantially restricted with the non-singing masks, especially the N95 respirator. Three repeats were performed and error bars are 1 sigma geometric standard deviations. The reported emission rates have not been corrected for flow. (right axis) The associated reduction efficiency for exhaled particles sampled in the forward (through mask) direction (black points). 


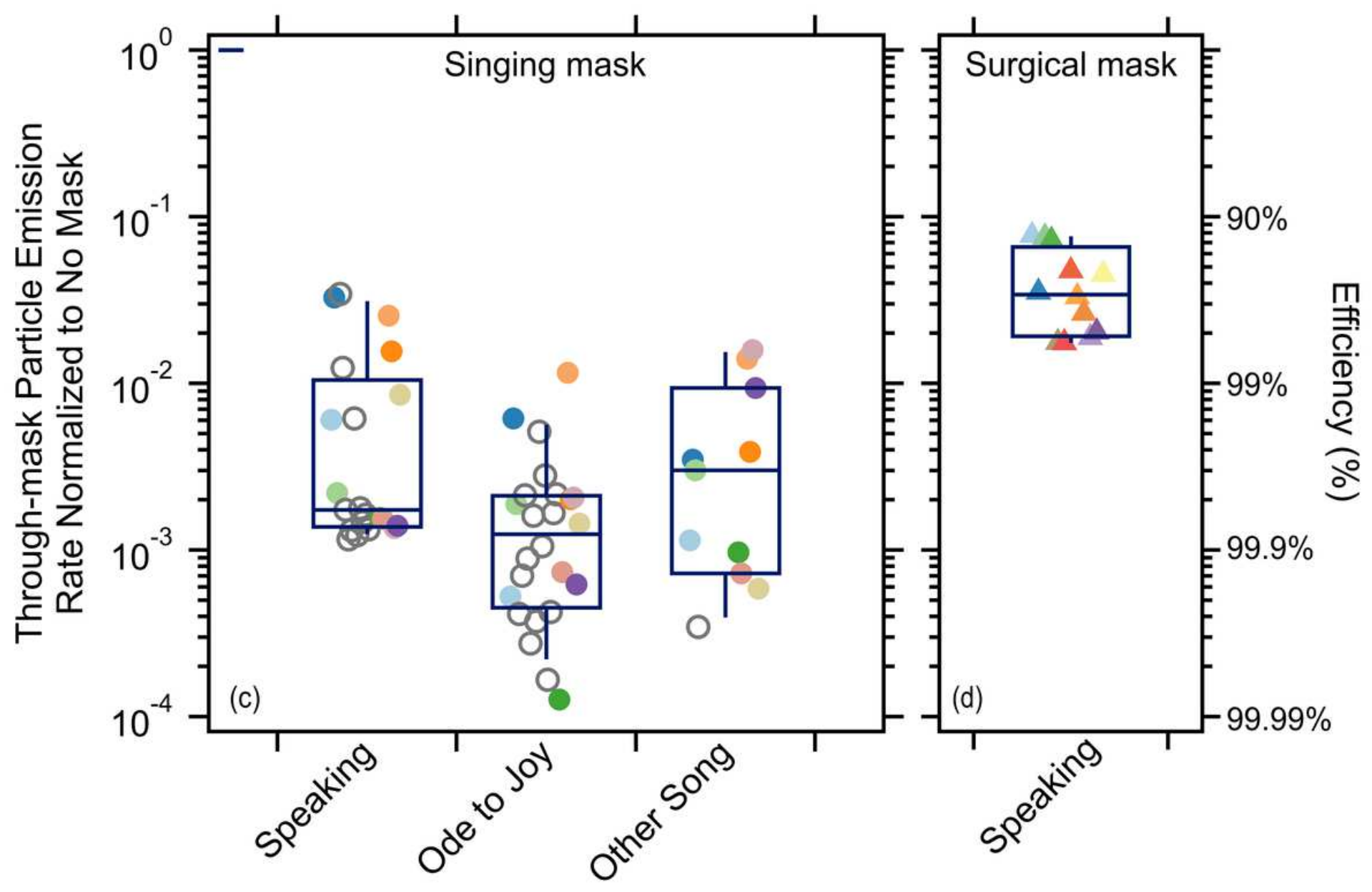

Figure 4

(a) The through-mask (forward) particle emission rates normalized to no mask (left axis) or the corresponding reduction efficiency (right axis) observed for participants while wearing the singing mask for speaking or singing either the Ode to Joy or a song chosen by the participant. Closed colored circles in (a) indicate unique individuals while open gray circles indicate repeats by one individual. (b) Normalized particle emission rates for participants speaking while wearing a surgical mask (original data from Cappa et al. [2021]). Note that the participants in (a) differ from those in (b). Box and whisker plots show the median, 25th/75th percentile, and 10th/90th percentile. 


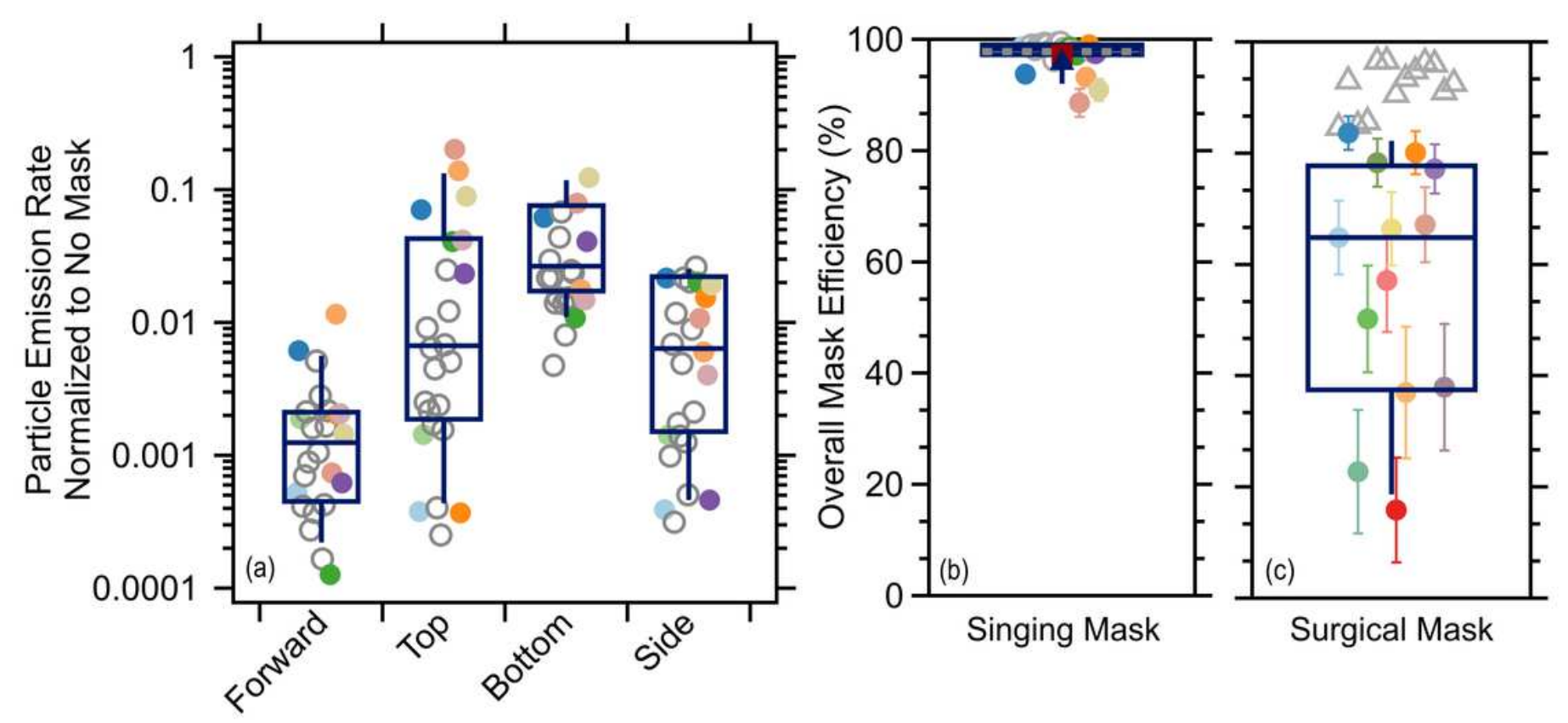

Figure 5

(a) Particle emission rates normalized to no mask wearing for singing Ode to Joy for the different orientations while wearing the singing mask. (b) The overall mask efficiency while singing Ode to Joy with the singing mask, accounting for leakage flows, colored by participant (circles). Uncertainty bars on individual points indicate $1 \rrbracket$ determined from Monte Carlo simulations. The red square is the average across all measurements and the blue triangle the average after combining replicate measurements from one participant. Closed circles in (a) and (b) indicate unique individuals while open circles indicate repeats by one individual. (c) Overall efficiency for speaking with a surgical mask including leakage flows (solid points) or assuming all flow passes through the mask, i.e., zero leakage flows and perfect sealing (open points). Original data from Cappa et al. [2021], but reprocessed here for individuals. Note that the participants in $(a, b)$ differ from those in (c). Box and whisker plots show the median, 25th/75th percentile, and 10th/90th percentile. 


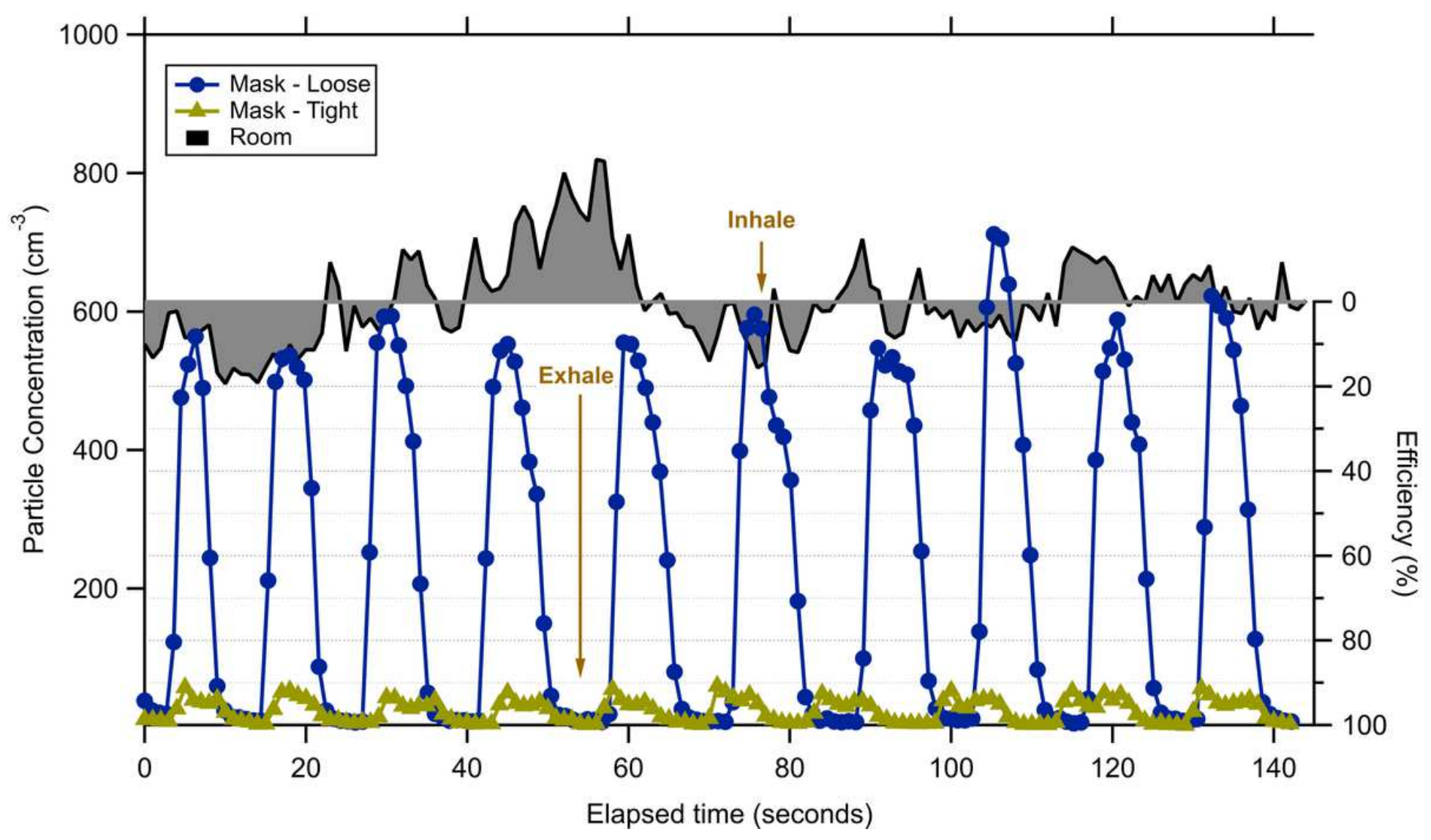

Figure 6

Time-series of particle concentrations measured using a CPC for room air (solid black line), sampled inside the singing mask worn intentionally loose around the neck (blue circles), and when sampled inside the mask when worn tightly as designed (gold triangles). The right axis shows the approximate mask efficiency based on comparison with the mean room air concentration. 

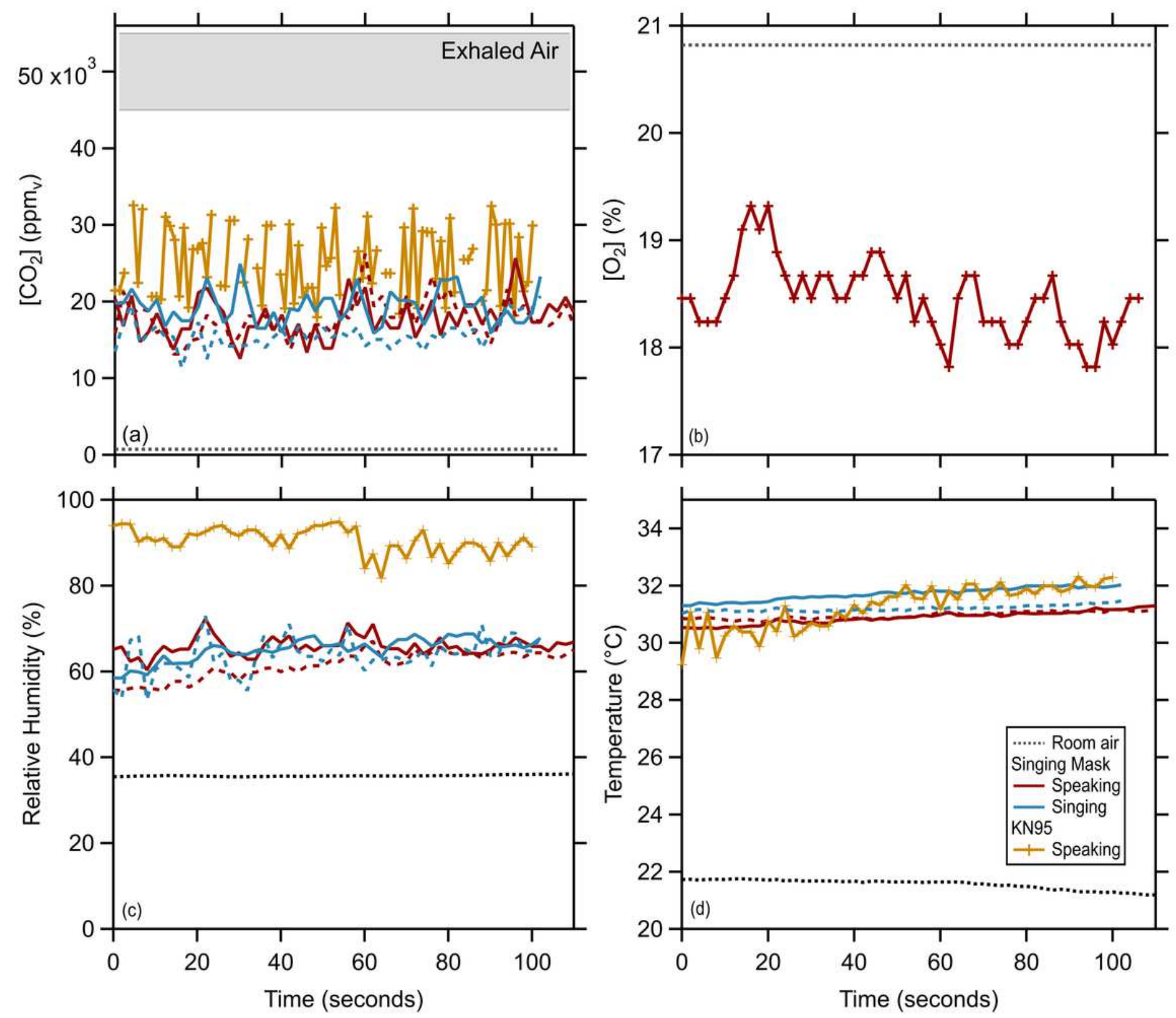

Figure 7

(a) $\mathrm{CO} 2$ and (b) 02 concentrations along with (c) relative humidity and (d) temperature measured in the deadspace of the singing mask during speaking (red) and singing (blue) and in the deadspace of a KN95 respirator during speaking (gold), compared with that for room air (black line) and in typical exhaled air (gray band). Dashed versus solid lines differentiate replicates.

\section{Supplementary Files}

This is a list of supplementary files associated with this preprint. Click to download.

- OperaMasksR3SuppMatFinal.pdf 